\title{
A NEW METHOD FOR THE DIRECT MEASUREMENT OF SPECTRAL LINE STRENGTHS AND WIDTHS*†
}

\author{
R. E. MEREDITH \\ Willow Run Laboratories, Institute of Science and Technology, The University of Michigan, Ann Arbor, U.S.A.
}

(Received 3 June 1971)

\begin{abstract}
The most important sources of error incurred in the measurements of spectral-line parameters arise from uncertainty in the determination of the 100 per cent transmittance and in the distortion of the line profile by the spectrometer. These errors have been investigated numerically by passing an idealized spectrometer slit function over several assumed line profiles. In this way, families of correction curves have been constructed, from which spectral-line strengths, widths, and peak absorption coefficients may be determined from apparent values measured directly from the chart recorder. The effect of the form of the slit function has been investigated by using triangular, Gauss, Cauchy and combination Gauss-Cauchy slit functions. The effect of uncertainties in the line shape has been investigated by using Doppler, Lorentz, and Voigt line shapes.

The correction procedure has been applied to the self- and nitrogen-broadened $R(0)$ and $P(1)$ lines in the first overtone band of hydrogen fluoride. The measurements were performed on collision-broadened lines near the linear region of growth; within experimental error, the line parameters were found to obey the Lorentz relation $k^{P}=S / \gamma \pi$. The measured self-broadened line widths indicate a non-Lorentz behavior because they do not vary linearly with pressure. When broadened by nitrogen, the line widths follow the expected linear dependence on pressure, well within experimental error.
\end{abstract}

\section{INTRODUCTION}

SPECTRAL line-shape measurements may be separated into two types. On the one hand, there are measurements which try to define detailed information about the true line shape. On the other hand, once the true shape is already known, there are measurements which try to define only spectral line-widths and strengths. The present investigation treats only the latter type, though it is clear that some line-profile information may be obtained by inference. Our primary goal is the development of a procedure to determine line strengths and widths which makes maximum use of the moderate- to high-resolution capability now available in most spectroscopic laboratories, and which may be applied with relative ease, without regard to weak continuum absorption or emission superimposed on the line spectrum.

Often the attitude is taken that the accuracy of measured line parameters is relatively independent of the spectral resolution with which observations are made. This feeling is

* This research was sponsored by the Advanced Research Projects Agency under ARPA Order 236, Amendment 38, Contract DAHC15-67-C-0062.

† In what follows, only absorptance measurements will be discussed. The methods developed may be used for measurements of radiant intensity profiles as well. 
particularly common in the case of absorption* line-strength measurements, since it has been shown both theoretically and experimentally that the area under an absorptance line (i.e. the equivalent width) is independent of instrumental distortion. ${ }^{(1,2)}$ On the other hand, one intuitively feels that, with greater spectral resolution, more information is obtained. Thus, one is confronted with an apparent paradox. However, the resolution of this paradox is immediate upon recognition of the fact that the major source of error in measurements of integrated absorptance is the area lost (or gained) by inaccurate location of the 100 per cent transmittance lines. This error arises from the difficulty in measuring absorptance values which are less than several percent. It is a limitation imposed by photometric stability rather than by spectral resolution. A more accurate measurement of equivalent width is possible if most of the absorptance is concentrated near the line center and if it diminishes rapidly within several half widths of the center, thereby minimizing the lost area. The use of high resolution alleviates this limitation in two ways: (1) measurement is limited only by stability rather than by slit-induced wing absorption which increases the lost area, (2) observation is possible for Voigt or Doppler profiles (which have a very small wing contribution), rather than for collision-broadened lines which have an appreciable contribution far from the line center. In addition, the use of high resolving power to eliminate or minimize the wing absorption may eliminate the problem of line overlap and result in more meaningful measurements.

The importance of resolution in the determination of line widths is much more obvious. It is worth pointing out, however, that in order to obtain independent measurements of line strengths and widths, resolution sufficient for the observation of relatively weak lines must be used, since the analytical connection between line strength and width is greatest for strong lines. In the weak-line limit, the strength depends on width only through the wing contribution.

A significant step forward in the attempt to maximize the information contained in moderate- to high-resolution measurements was achieved with the introduction of direct measurement by KosTKowsKI and BASs. ${ }^{(3)}$ Their method is essentially a parametric slit correction procedure which may be applied for both line strength and width measurement if the instrumental distortion is not large (i.e. if the ratio of spectral slit width to line half width is of order unity). The direct measurement procedure has removed most of the limitations of the equivalent width method, but still leaves two major sources of error: the errors associated with the determination of the line of 100 per cent transmittance (the base line), and the errors arising from the contribution of the wings of distant lines. One of the main objectives of this investigation is the removal of these two sources of error from the direct measurement method.

\section{THE DIRECT MEASUREMENT MFTHOD}

The mathematical expression describing the distortion of a spectral absorptance line profile $\alpha(v)$ by a spectrometer which has a slit function $\sigma\left(v, v^{\prime}\right)$ may be written as follows:

$$
\alpha_{0}\left(v^{\prime}\right)=\frac{\int_{-\infty}^{+\infty} \sigma\left(v, v^{\prime}\right) \alpha(v) \mathrm{d} v}{\int_{-\infty}^{+\infty} \sigma\left(v, v^{\prime}\right) \mathrm{d} v}=\frac{\int_{-\infty}^{+\infty} \sigma\left(v, v^{\prime}\right)\{1-\exp [-k(v) l]\} \mathrm{d} v}{\int_{-\infty}^{+\infty} \sigma\left(v^{\prime}, v^{\prime}\right) \mathrm{d} v} .
$$

* In what follows, only absorptance measurements will be discussed. The methods developed may be used for measurements of radiant intensity profiles as well. 
Numerical integration of the integrals in equation (1) will yield the absorptance $\alpha_{0}\left(v^{\prime}\right)$ expected at wavenumber $v^{\prime}$. If $\alpha\left(v^{\prime}\right)$ and $\sigma\left(v, v^{\prime}\right)$ are precise descriptions of a line profile and the instrument function used to observe it, then

$$
\alpha_{0}(v) \equiv \alpha_{\text {obs }}(v)
$$

where $\alpha_{\mathrm{obs}}(v)$ is the absorptance observed experimentally. Equation (2) is the assumption on which the direct measurement method is based. A set of expected and observed line parameters may be defined in analogy with Lambert's law as follows:

$$
\begin{aligned}
\alpha_{0}(v) & =1-\exp \left[-k_{0}(v) l\right] \\
\alpha_{\mathrm{obs}}(v)= & 1-\exp \left[-k_{\mathrm{obs}}(v) l\right] \\
S_{0} & =\int_{-\infty}^{+\infty} \frac{k_{0}(v)}{p} \mathrm{~d} v \\
S_{\mathrm{obs}} & =\int_{-\infty}^{+\infty} \frac{k_{\mathrm{obs}}(v) \mathrm{d} v}{p} \\
k_{0}\left(v=\gamma_{0}\right)= & \frac{k_{0}^{P}}{2} \\
k_{\mathrm{obs}}\left(v=\gamma_{\mathrm{obs}}\right)= & \frac{k_{\mathrm{obs}}^{P}}{2}
\end{aligned}
$$

where the strengths $S_{0}$ and $S_{\text {obs }}$ have units (atmosphere $\left.\mathrm{cm}^{2}\right)^{-1}$, and $k_{0}^{P}$ and $k_{\mathrm{obs}}^{P}$ are the values of the expected and observed absorption coefficient at line center. Equations (1), (2) and (3) form the link between the unknown absorption coefficient $k(v)$ and $k_{\text {obs }}(v)$ measured directly from the chart recorder. This link is expressed in the form of tables or curves relating correction factors $\gamma_{0} / \gamma, k^{P} / k_{0}^{P}$, and $S / S_{0}$ to the degree of instrumental distortion, $2 a / \gamma$, where the quantity $a$ is the half width at half height of $\sigma\left(v, v^{\prime}\right)$ and where $\gamma, \gamma_{0}$, and $\gamma_{\text {obs }}$ are the half widths at half height of $k(v), k_{0}(v)$, and $k_{\text {obs }}(v)$. A typical correction curve for a Lorentz line which has a peak absorption $\alpha^{P}=15$ per cent and a Gauss slit function,

$$
\begin{aligned}
k_{L}(v) & =\frac{S \gamma p}{\pi\left[\left(v-v_{0}\right)^{2}+\gamma^{2}\right]} \\
\sigma\left(v, v^{\prime}\right) & =\frac{1}{a \sqrt{ } \pi} \exp \left[\frac{\left(v-v^{\prime}\right)^{2}}{a^{2}}\right]
\end{aligned}
$$

is shown in Fig. 1.

In order to determine the half width of a Lorentz line by the direct measurement method, two measurements of a given line are required, since the ratio $2 a / \gamma$ must be determined. For example, if measurements $\gamma_{\mathrm{obs}}(2 a)$ and $\gamma_{\mathrm{obs}}(4 a)$ are made with spectral slit widths $2 a$ and $4 a$, Fig. 2 may be used to deduce the value of $2 \alpha / \gamma$ to be used to correct $\gamma_{\text {obs }}(2 a)$. A detailed discussion of the method may be found in Refs. 3 and 4. 


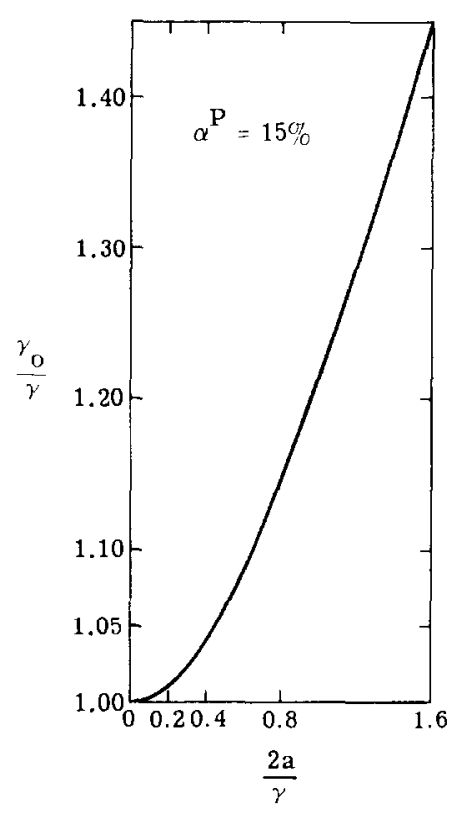

FIG. 1. A plot of correction factor $\gamma_{c}=\gamma_{0} / \gamma$ for the determination of half-widths $\gamma$.

\section{A MODIFIED DIRECT MEASUREMENT METHOD}

The direct measurement method suffers from the same major source of error inherent in all absolute intensity measurements : the inaccuracy inherent in the determination of the 100 per cent transmittance deflection. This is the error depicted by the shaded portion of

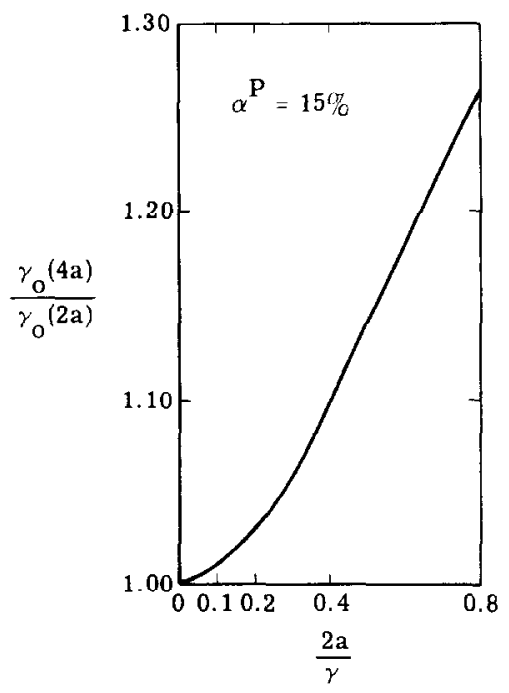

FIG. 2. The relation between the ratio $2 a / \gamma$ and two expected half-widths $\gamma_{0}$ for slit functions of width $2 a$ and $4 a$. 
Fig. 3. The following discussion describes a method by which this error may be avoided in the direct measurement of line parameters.

Suppose an expected absorptance curve is computed numerically from equation (1). If a straight line of height $\alpha_{0}(G)$ above the true 100 per cent transmittance is drawn extending $\pm G$ half widths from the line center, as shown in Fig. 4, one would have the expected absorptance curve which corresponds to a measurement with a spectrometer having the specified slit function and $2 a / \gamma$ ratio, but which differs by the shaded portion of Fig. 3. This portion is assumed to be the actual area and height lost by the error in the determination of the 100 per cent transmittance. A slightly altered expected absorptance curve $\alpha_{0}^{\prime}(v)$ can be calculated using this new 100 per cent line by simply subtracting the height $\alpha_{0}(G)$ from the absorptance points $\alpha_{0}(v)$ and replacing $\tau(100$ per cent $)$ by $\tau[100$ per cent $\left.-\alpha_{0}(G)\right]=\tau^{\prime}(100$ per cent $)$. This is the lower dashed curve of Fig. 4 ; the corresponding absorption coefficient is the upper dashed curve. This $k_{0}^{\prime}(v)$ is an even poorer representation of the actual absorption coefficient $k(v)$ than the $k_{0}(v)$ defined earlier, because its apparent area, width and peak is smaller. However, since correction curves can be constructed and the true line parameters determined as before, the quality of the representation is of little consequence. The essential difference lies in the existence of a set of curves or correction tables for each distance $G$ to which the measurement is made from the line center. Once the true line width $\gamma$ is known, $G$ is determined, and the appropriate correction to $S_{\text {obs }}$ and $k_{\mathrm{nbs}}^{P}$ may be made.

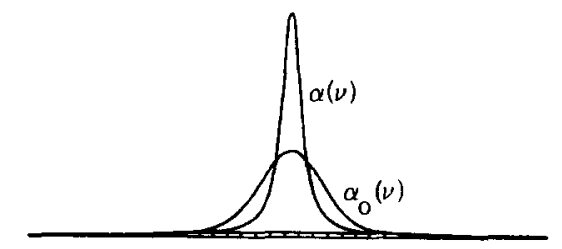

FIG. 3. Effect of errors in baseline determination.

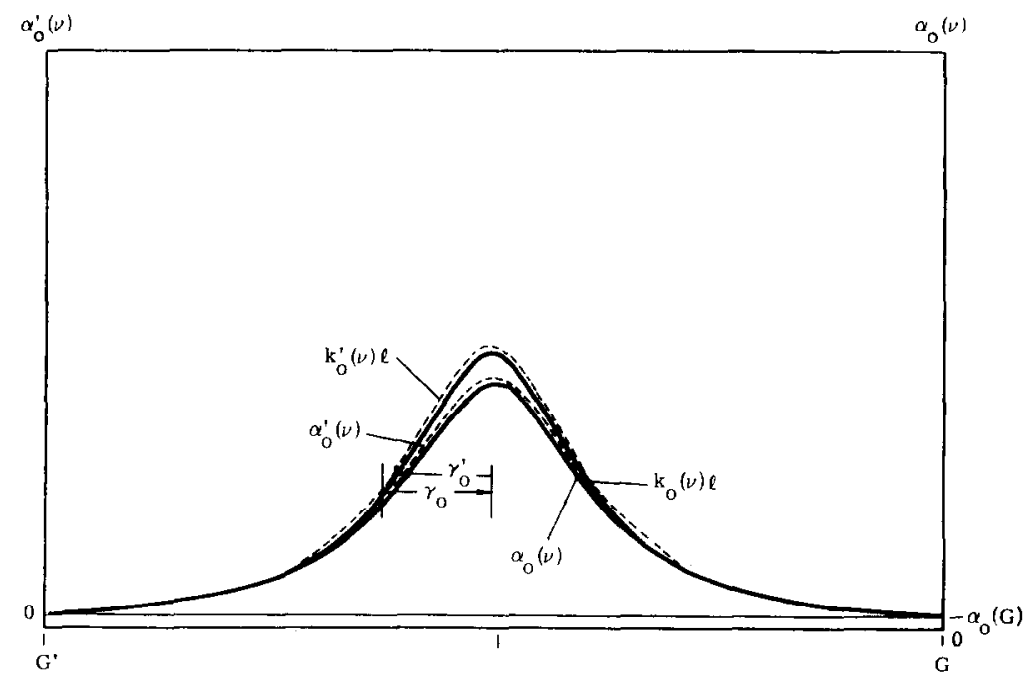

Fig. 4. The determination of the artificial absorptance curve $\alpha_{0}^{\prime}(v)$ from $\alpha_{0}(v)$. 
Appendix I gives the corrections to a Lorentz line having a variety of true peak absorptances for several ratios $2 a / \gamma$ and for a number of distances $G$ into the line wings. Figs. 5-7 show families of correction curves for $\alpha^{p}=30$ per cent. Although these curves are quite similar to the familiar $G=\infty$ curves discussed previously, there is an obvious difference. It can be seen that as $2 a / \gamma \rightarrow 0$, the correction curve approaches a nonzero value. This zero point correction arises from the wing correction and from the rectangle of height, $\alpha_{0}(G)$, and length, $2 G \gamma$, that was lost in drawing the 100 per cent line. As $G \rightarrow \infty$, it can be seen that

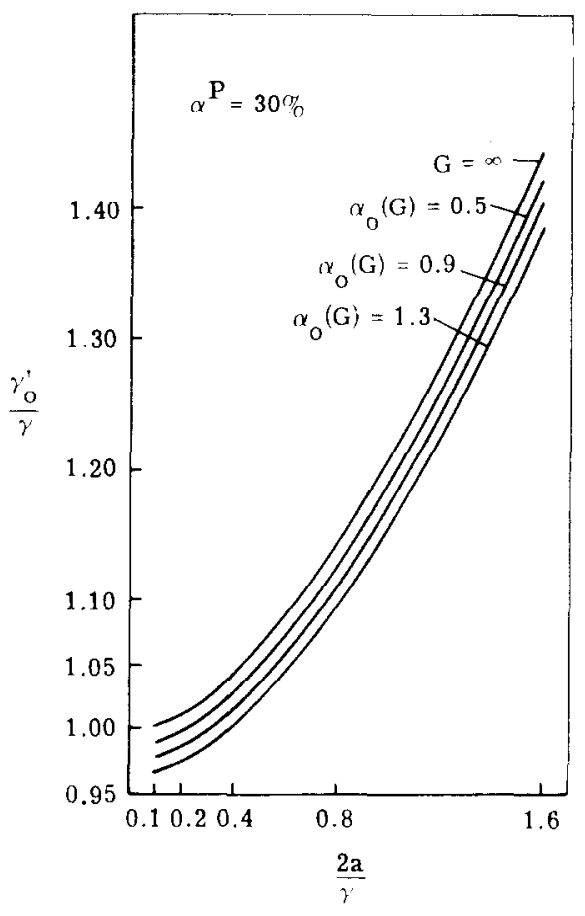

FIG. 5. Correction factor $\gamma_{\mathrm{c}}$ for $\gamma_{\mathrm{obs}}$ for measurements taken to $G$.

this zero point correction approaches zero and that the curves are the same as those discussed earlier.* The wing corrections are thus contained naturally in the present method.

It can be seen also that the $\gamma_{0}^{\prime} / \gamma$ correction curves show negative corrections for small $G$ at small $2 a / \gamma$. This is to be expected, since small $G$ corresponds to large $\alpha_{0}(G)$, a drastic raising of the apparent 100 per cent transmittance. $\gamma_{0}^{\prime}$ can actually be smaller than $\gamma$ for sufficiently small $G$, since it raises the half height of the line well above the true half height. This does not occur for relatively large $2 a / \gamma$ because the distortion of the line is sufficient to compensate somewhat for the effect of the raised base line.

From the $\gamma_{0}^{\prime} / \gamma$ versus $2 a / \gamma$ correction curves, curves $\gamma_{0}^{\prime}(4 a) / \gamma_{0}^{\prime}(2 a)$ versus $2 a / \gamma$ may be constructed from Fig. 5 with the use of curves of constant $G$. In fact, if $2 a / \gamma$ is not too large

* The corrections to $\gamma_{\mathrm{ob},}, k_{\mathrm{obs}}^{P}$, and $S_{\mathrm{obs}}$ computed here are not in exact agreement with the results of Kostkowski and Bass. This discrepancy, though small, is significant for very accurate measurements. The present work is in complete agreement with that of IZATT, ${ }^{(5)}$ who extended the Kostkowski and Bass curves to larger values of $\alpha$. 


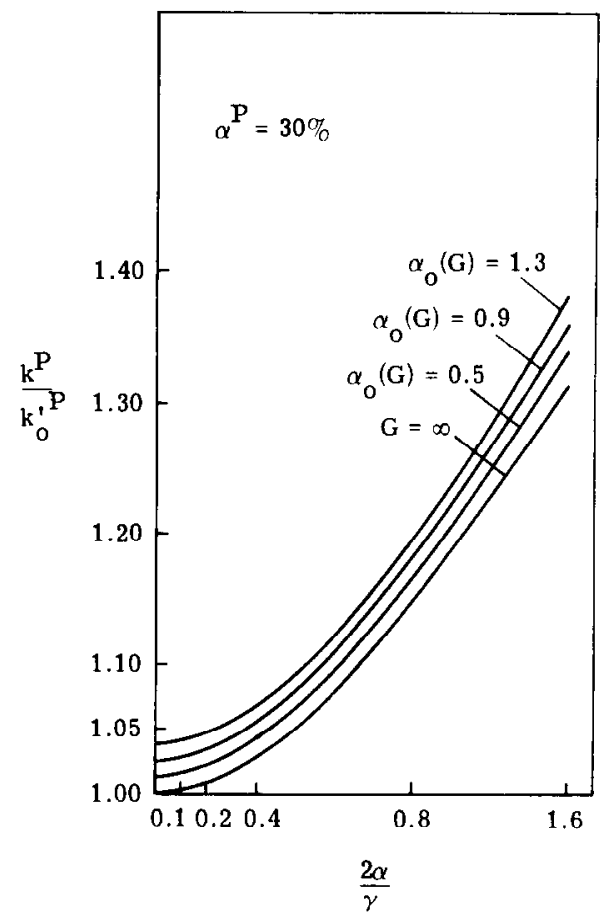

F1G. 6. Correction factor $K_{c}^{P}$ for $K_{\text {obs }}^{P}$ for measurements taken to $G$.

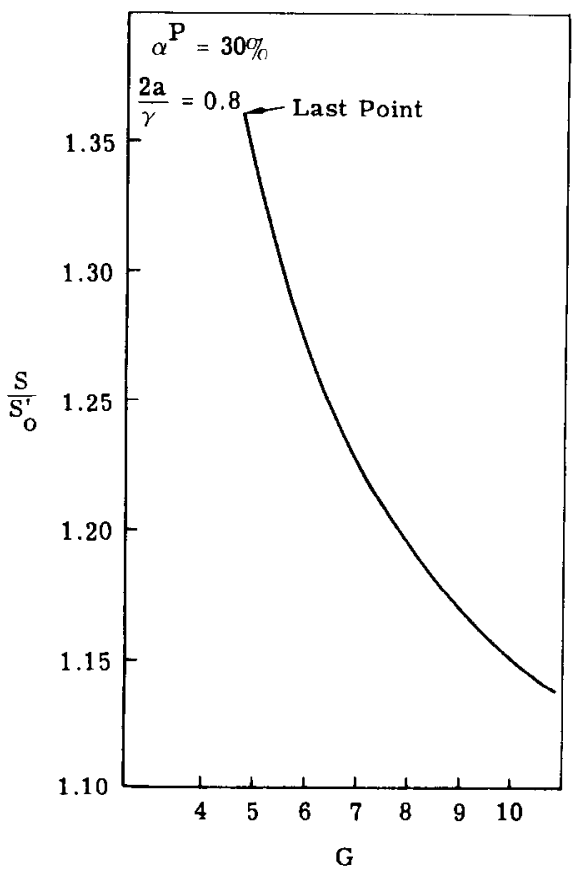

FiG. 7. Correction factor $S_{c}$ for $S_{\text {obs }}$ for measurements taken to $G$. 
and if $G$ is not too small, the latter curves are independent of $G$; i.e. if the measurement is made a distance $G$ beyond which the distortion is small, the $\gamma_{0}^{\prime}(4 a) / \gamma_{0}^{\prime}(2 a)$ versus $2 a / \gamma$ curves of finite $G$ are the same as the $G=\infty$ curves. In Table 1 , values of $\gamma_{0}^{\prime}(4 a) / \gamma_{0}^{\prime}(2 a)$ versus $2 a / \gamma$ for a wide range of $G$ are listed. It is clear from this table that all reasonable $G$ give a unique curve at or near the linear region of growth. For stronger lines, this is no longer the case, and care must be exercised that the correct curve is used for the determination of $2 a / \gamma$. It should be emphasized that this method requires (1) that the two sets of measurements be made out to equal numbers of half widths from the line center, and (2) that this distance extend to a region of small instrumental distortion.

Since the ratio $\gamma_{0}^{\prime}(4 a) / \gamma_{0}^{\prime}(2 a)$ has a negligible dependence on $\alpha^{P}$, the value $2 a / \gamma$ may be obtained without a prior knowledge of $\alpha^{P}$. Also, since the correction curves depend only weakly on $\alpha^{P}$, the proper choice of $\alpha^{P}$ may be made quickly by iteration.

\begin{tabular}{ccccc}
\hline \multicolumn{5}{c}{$\alpha_{0}^{\prime}(G)$} \\
\hline$\gamma$ & 0 & \multicolumn{5}{c}{0.3} & 0.9 & 1.3 \\
\hline \multicolumn{5}{c}{$\alpha^{P}=30 \%$} \\
0.1 & 1.0077 & 1.0073 & 1.0075 & 1.0075 \\
0.2 & 1.0287 & 1.0290 & 1.0285 & 1.0282 \\
0.4 & 1.0986 & 1.0982 & 1.0977 & 1.0978 \\
0.8 & 1.2650 & 1.2638 & 1.2616 & 1.2599 \\
1.6 & 1.5059 & 1.5015 & 1.4926 & 1.4865
\end{tabular}

\begin{tabular}{lclcc} 
& 0 & \multicolumn{1}{c}{0.5} & 1.1 & 1.5 \\
\cline { 3 - 3 } & \multicolumn{5}{c}{$\alpha^{p}=60 \%$} \\
0.1 & 1.0070 & 1.0068 & 1.0063 & 1.0067 \\
0.2 & 1.0263 & 1.0264 & 1.0263 & 1.0261 \\
0.4 & 1.0920 & 1.0917 & 1.0914 & 1.0912 \\
0.8 & 1.2576 & 1.2566 & 1.2556 & 1.2546 \\
1.6 & 1.5071 & 1.5039 & 1.5000 & 1.4971
\end{tabular}

\begin{tabular}{llrl} 
& 0 & 1.1 & 1.7 \\
\cline { 3 - 4 } 0.1 & \multicolumn{3}{c}{$\alpha^{P}=90 \%$} \\
0.2 & 1.0051 & 1.0049 & 1.0049 \\
0.4 & 1.0203 & 1.0202 & 1.0201 \\
0.8 & 1.0779 & 1.0775 & 1.0772 \\
1.6 & 1.2487 & 1.2473 & 1.2465 \\
\hline
\end{tabular}

\section{APPLICATION TO THE $R(0)$ AND $P(1)$ LINES IN THE FIRST OVERTONE BAND OF HYDROGEN FLUORIDE}

The new procedure developed here for the correction of slit effects is best illustrated by its application to isolated line profiles observed in the laboratory. For this purpose, the method will be applied to the $R(0)$ and $P(1)$ lines in the first overtone band of hydrogen fluoride. 
Absorptance measurements on these lines have been carried out for path lengths of $0.470 \mathrm{~cm}, 0.498 \mathrm{~cm}$ and $1.015 \mathrm{~cm}$ in the case of self-broadened $\mathrm{HF}$ and at path lengths of $1.99 \mathrm{~cm}$ and $7.50 \mathrm{~cm}$ in the case of $\mathrm{HF}$ mixed with nitrogen in a ratio of 1 part $\mathrm{HF}$ to 25 parts $\mathrm{N}_{2}$. Self-broadened lines were observed for pressure of $\frac{1}{2}$ and $\frac{1}{4}$ standard atmosphere, and the $\mathrm{N}_{2}$-broadened lines were observed for total pressures of approximately one and two atmospheres. All measurements were carried out with absorption cell temperatures at about $100^{\circ} \mathrm{C}$. Complete experimental details will be described in a later paper.

For one particular path length, sets of measurements at two spectral slit widths ( $2 a$ and $4 a$ ) were taken. At least five 'good' scans were made of each line at a given slit setting (a 'good' scan is defined as one which appears to be undisturbed by source or detection fluctuations or by environmental effects). After each scan, an opaque shutter was inserted in the optical path to determine the zero line (the recorder deflection corresponding to zero transmittance). A background was scanned for each line for the exact conditions of the actual measurement. The direct measurement of the observed peak absorption coefficient, Lorentz half width, and line strength for both sets of measurements was carried out in the following steps :

(1) The zero line was adjusted to account for the chopped scattered light (i.e. the impure radiation) striking the detector. This amounted to 0.8 per cent for both the $R(0)$ and $P(1)$ lines.

(2) A base line was drawn for each line extending to the extreme wings. This base line can be considered only an approximation to the true 100 per cent transmittance, the determination of which is possible by evacuation of the absorption cell after each scan.

(3) An arbitrary number of half widths $G$ from line center was chosen and an adjusted base line drawn for each line. This base line was necessarily a fraction of a percent or more above the original. The value $G$ was chosen so that it was the largest value compatible with all lines of both slit settings, but a small enough value to connect the absorptance contour as it approached the original base line most accurately.

(4) The zero line, base line, and line contour were recorded on data-processing cards for determining the percentage of absorptance, the observed absorption coefficient, and their areas (in units of wavenumber).

(5) The peak value of the observed absorption coefficient and its half width at half height were determined. This procedure gave values $S_{\mathrm{obs}}^{\prime}, k_{\mathrm{obs}}^{P^{\prime}}$ and $\gamma_{\mathrm{obs}}^{\prime}$ for each line having the same value, $G \gamma$.

(6) Values $\gamma_{\text {obs }}^{\prime}(2 a)$ and $\gamma_{\text {obs }}^{\prime}(4 a)$ were determined by an averaging process, and the ratio $\gamma_{\text {obs }}^{\prime}(4 a) / \gamma_{\text {obs }}^{\prime}(2 a)$ was used to determine the ratio $2 a / \gamma$ from Table 1 . This $2 a / \gamma$ value was used in correction curves such as Figs. 5-7 to determine the corrections to $\gamma_{\mathrm{abs}}^{\prime}(2 a), \gamma_{\mathrm{obs}}^{\prime}(4 a)$ $k_{\mathrm{obs}}^{P \prime}(2 a), k_{\mathrm{obs}}^{P \prime}(4 a), S_{\mathrm{obs}}^{\prime}(2 a)$, and $S_{\mathrm{obs}}^{\prime}(4 a)$. The proper correction curve within each family of curves was determined by the use of the known value of slit width, $2 a$, to determine a first approximation to $\gamma$ with the use of the ratio $2 a / \gamma$. This value of $\gamma$ is necessary to determine the number of true half widths in the distance $G$ from the line center. Table 2 shows the results for self-broadened HF lines.

In an effort to reduce error in the final strength and width values, the $1.015-\mathrm{cm}$ and $0.47-\mathrm{cm}$ path-length measurements were carried out for the narrower slit setting. Table 2 also shows the results of these measurements. In all cases, the value of $\gamma$, determined from the $l=0.498 \mathrm{~cm}$ data, was used to find the corrections $S_{c}, \gamma_{c}$, and $K_{c}^{P}$. Table 3 gives the actual measurcd quantities and the corrections used in arriving at the values given in Table 2. 
TABLE 2. LINE PARAMETERS FOR SELF-BROADENED HF

\begin{tabular}{lccccc}
\hline $\begin{array}{c}p \\
\text { Line }\end{array}$ & $\begin{array}{c}l \\
(\mathrm{~cm})\end{array}$ & $k^{P l}$ & $\begin{array}{c}\hat{\gamma} \\
\left(\mathrm{cm}^{-1}\right)\end{array}$ & $\begin{array}{c}S \\
\left(\mathrm{~atm} \mathrm{~cm}^{2}\right)^{-1}\end{array}$ \\
\hline$P(1)$ & $\frac{1}{2}$ & 0.498 & 0.228 & 0.229 & 0.650 \\
$P(1)$ & $\frac{1}{4}$ & 0.498 & 0.2175 & 0.126 & 0.672 \\
$P(1)$ & $\frac{1}{2}$ & 1.015 & 0.474 & 0.228 & 0.677 \\
$P(1)$ & $\frac{1}{4}$ & 1.015 & 0.414 & 0.130 & 0.690 \\
$P(1)$ & $\frac{1}{4}$ & 0.47 & 0.201 & 0.122 & 0.648 \\
$R(0)$ & $\frac{1}{2}$ & 0.498 & 0.288 & 0.202 & 0.749 \\
$R(0)$ & $\frac{1}{4}$ & 0.498 & 0.274 & 0.111 & 0.774 \\
$R(0)$ & $\frac{1}{2}$ & 1.015 & 0.588 & 0.204 & 0.732 \\
$R(0)$ & $\frac{1}{4}$ & 1.015 & 0.547 & 0.113 & 0.753 \\
$R(0)$ & $\frac{1}{4}$ & 0.47 & 0.257 & 0.108 & 0.728 \\
\hline
\end{tabular}

Aside from the good agreement among the many different measurements, the most striking feature of the measurements is the apparent nonlinear pressure dependence of the half widths $\gamma$. This seems to indicate that the lines are not really Lorentzian, as was assumed in using the direct measurement procedure. Nevertheless, the half widths and peak absorption coefficients are related to the line strength by the Lorentz expression $k^{P}=S p / \gamma \pi$, as may be verified for any of the parameters listed in Table 2. The lower pressure lines, for which the half width per unit of pressure is larger, have smaller values $k^{P}$, just sufficiently smaller, within experimental error, to obey the Lorentz relationship. The conclusion that must be drawn is that the self-broadened $R(0)-P(1)$ lines arc indeed Lorentzian near the

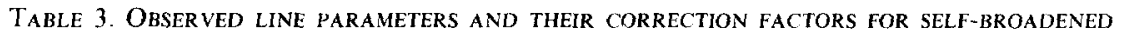
$R(0)$ AND $P(1)$ LINES IN THE $v=0 \rightarrow 2$ BAND OF HF

\begin{tabular}{lllllllll}
\hline \multicolumn{1}{c}{ Slit } & $2 a$ & $4 a$ & $2 a$ & $4 a$ & $2 a$ & $2 a$ & $2 a$ \\
\hline$l$ & 0.498 & 0.498 & 0.498 & 0.498 & 1.015 & 1.015 & 0.47 \\
$p$ & 0.501 & 0.501 & 0.253 & 0.253 & 0.471 & 0.250 & 0.252 \\
$G ;$ & 1.005 & 1.005 & 0.567 & 0.567 & 1.385 & 0.756 & 0.635 \\
$\gamma_{\text {obs }}$ & 0.206 & 0.240 & 0.128 & 0.168 & 0.197 & 0.131 & 0.126 \\
$\gamma_{c}^{p}$ & 1.022 & 1.179 & 1.15 & 1.49 & 1.05 & 1.165 & 1.15 \\
$k_{\mathrm{obs}}^{P}$ & 0.263 & 0.240 & 0.233 & 0.187 & 0.562 & 0.4672 & 0.228 \\
$k_{\mathrm{c}}^{P}$ & 1.09 & 1.21 & 1.175 & 1.47 & 1.07 & 1.17 & 1.18 \\
$S_{\text {obs }}^{P}$ & 0.571 & 0.571 & 0.599 & 0.599 & 0.603 & 0.616 & 0.564 \\
$S_{\mathrm{c}}$ & 1.338 & 1.34 & 1.335 & 1.34 & 1.22 & 1.235 & 1.29
\end{tabular}

\begin{tabular}{llllllll}
\multicolumn{1}{c}{ Slit } & $2 a$ & $4 a$ & $2 a$ & $4 a$ & $2 a$ & $2 a$ & $2 a$ \\
\hline$l$ & 0.498 & 0.498 & 0.498 & 0.498 & 1.015 & 1.015 & 0.47 \\
$p$ & 0.501 & 0.501 & 0.253 & 0.253 & 0.471 & 0.250 & 0.252 \\
$g$ & 1.04 & 1.04 & 0.616 & 0.616 & 1.48 & 0.740 & 0.616 \\
$\gamma_{\text {obs }}$ & 0.230 & 0.256 & 0.1383 & 0.175 & 0.219 & 0.149 & 0.138 \\
$\gamma_{c}^{\text {p }}$ & 1.00 & 1.105 & 1.10 & 1.39 & 1.03 & 1.10 & 1.11 \\
$k_{\text {obs }}^{\text {l }}$ & 0.208 & 0.196 & 0.189 & 0.156 & 0.454 & 0.357 & 0.190 \\
$k_{c}^{P}$ & 1.08 & 1.165 & 1.15 & 1.38 & 1.06 & 1.14 & 1.15 \\
$S_{\text {obs }}^{\text {s }}$ & 0.460 & 0.460 & 0.485 & 0.485 & 0.542 & 0.525 & 0.532 \\
$S_{c}$ & 1.39 & 1.39 & 1.36 & 1.37 & 1.22 & 1.30 & 1.34 \\
\hline
\end{tabular}


line center, with strengths 0.741 and 0.670 , respectively, but that their half widths per unit of pressure are not constant. This is not inconsistent with the measurements of HERGET et $a l^{(6)}$ who determined that under certain conditions, self-broadened HF lines are nonLorentzian in the distant wings. The lines investigated here were sufficiently weak to assure that such an effect was not detected.

The results for the nitrogen-broadened $R(0)$ and $P(1)$ lines are given in Table 4. The measured strengths are in adequate agreement with the self-broadened values, and the scatter in the measured values indicates the precision with which the measurements were made. Once again the relationships among $k^{P}, \gamma$ and $S$ expected for Lorentz lines hold quite nicely. Unlike the self-broadened line widths, however, the nitrogen-broadened lines show a linear dependence on pressure. Therefore, the values $k^{P}$, are essentially the same for a given absorption path length, independent of pressure. Thus, it is tempting to conclude that spectral lines broadened by the nonpolar molecules are more Lorentzian than self broadened lines of strongly polar molecules. It is hoped that further foreign gasbroadening measurements will shed more light on this situation.

TABLE 4. LINE PARAMETERS FOR NITROGEN-BROADENED HF

\begin{tabular}{llllll}
\hline Line & $\begin{array}{c}p \\
(\mathrm{~atm})\end{array}$ & $\begin{array}{c}l \\
(\mathrm{~cm})\end{array}$ & $\left.k^{P}\right)$ & $\begin{array}{c}\gamma \\
\left(\mathrm{cm}^{-1}\right)\end{array}$ & $\begin{array}{c}S \\
\left(\mathrm{~atm} \mathrm{~cm}^{2}\right)^{-1}\end{array}$ \\
\hline$P(1)$ & 2 & 7.5 & 0.528 & 0.214 & 0.643 \\
$P(1)$ & 1 & 7.5 & 0.540 & 0.1081 & 0.660 \\
$P(1)$ & 1 & 1.99 & 0.140 & 0.106 & 0.652 \\
$R(0)$ & 2 & 7.5 & 0.632 & 0.209 & 0.740 \\
$R(0)$ & 1 & 7.5 & 0.636 & 0.106 & 0.763 \\
$R(0)$ & 1 & 1.99 & 0.162 & 0.104 & 0.771 \\
\hline
\end{tabular}

5. THE DOPPLER AND VOIGT LINE PROFILES

The Doppler absorption coefficient is given by

$$
k_{D}(v)=\sqrt{\left(\frac{\ln 2}{\pi}\right)} \frac{S}{\gamma_{D}} \exp \left[-\left(\frac{v-v_{0}}{\gamma_{D}}\right)^{2} \ln 2\right]
$$

where $\gamma_{D}$ is the half width at half height of $k_{D}(\gamma)$, and where the peak value of $k_{D}(v)$ is

$$
k_{D}^{P}=\frac{S}{\gamma_{D}} \sqrt{\left(\frac{\ln 2}{\pi}\right)} .
$$

Correction tables for expected absorptance profiles, $\alpha_{0 D}(v)$, are given in Appendix II.

If a spectral line is broadened by both the Doppler effect and collision damping, its absorption coefficient is given, to a good approximation, by the Voigt profile, as follows:

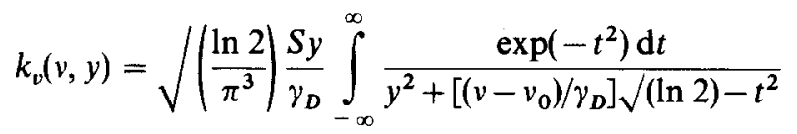


where $y=\left(\gamma_{L} / \gamma_{D}\right) \sqrt{ }(\ln 2)$. The peak value of $k_{v}(v, y)$ is given by

$$
k_{v}^{P}(y)=k_{v}(0, y)=\frac{S}{\gamma_{D}} \int\left(\frac{\log _{\mathrm{e}} 2}{\pi}\right) \exp \left(+y^{2}\right)[1-\operatorname{erf}(y)] .
$$

The half width of the Voigt profile $\gamma_{t}$, is not contained explicitly in equation (7), but it ranges between the values $\gamma_{v}=\gamma_{D}$ for $y=0$ and $\gamma_{v} \simeq \gamma_{L}$ for $\gamma_{D} \ll \gamma_{L}$. Correction tables for the Voigt profile are given in Appendix III for several values of $y$. The value of $2 a / \gamma_{t}$ may be determined by measurements at slit settings $2 a$ and $4 a$ as in the case of the Lorentz profile, and $y$ may be determined from a plot of $y$ versus $\gamma_{v} / \gamma_{D}$ (see Fig. 8) and from $\gamma_{D}$.

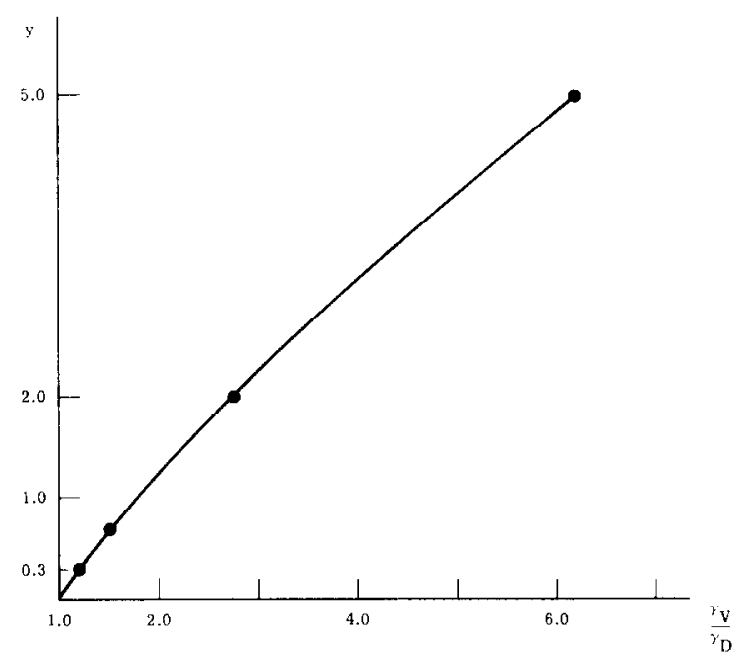

FIG. 8. Relation between $\gamma_{o}$ and $y=\sqrt{ }\left(\log _{\mathrm{e}} 2\right) \frac{\gamma_{L}}{\gamma_{D}}$.

\section{THE INFLUENCE OF THE FORM OF THE SLIT FUNCTION}

Often the slit function is more accurately described by an expression other than Gauss function. It is well known that a triangular slit function has much the same effect as the Gauss function, ${ }^{(3,7,8)}$ whereas an expression which has a slow falloff in the wings (e.g. the Cauchy function) results in a much more distorted spectrum. The present investigation into the effect of different slit functions has been limited to a quantitative comparison of the Gauss slit correction with the following slit functions:

(1) The triangular slit function

$$
\begin{array}{ll}
\sigma_{t}\left(v, v^{\prime}\right)=\frac{v^{\prime}-v}{4 a^{2}}+\frac{1}{2 a} & v^{\prime}-2 a \leq v \leq v^{\prime} \\
\sigma_{t}\left(v, v^{\prime}\right)=\frac{v-v^{\prime}}{4 a^{2}}+\frac{1}{2 a} & v^{\prime}<v<v^{\prime}+2 a .
\end{array}
$$

(2) The Cauchy function

$$
\sigma_{c}\left(v, v^{\prime}\right)=\frac{a^{2}}{\left(v-v^{\prime}\right)^{2}+a^{2}}
$$


(3) A combination Gauss-Cauchy function

$$
\begin{aligned}
& \sigma_{g q}\left(v, v^{\prime}\right)=\exp \frac{-\left(v-v^{\prime}\right)^{2} \ln 2}{a^{2}} \quad 0<\left|v-v^{\prime}\right|<a \\
& \sigma_{g q}\left(v, v^{\prime}\right)=\frac{a^{q}}{\left(v-v^{\prime}\right)^{q}+a^{q}} \quad a \leq v-v^{\prime} .
\end{aligned}
$$

If $q$ is four, $\sigma_{g q}$ falls off rapidly with $v-v_{0}$, and the slit corrections that result are quite similar to those of the Gauss function. For $q=2, \sigma_{g q}$ is very much like $\sigma_{c}$. Slit corrections for $\sigma_{t}, \sigma_{c}$, and $\sigma_{g 3}$ are presented in Appendices IV, V, and VI. A direct comparison of their effects on the direct measurement of a Lorentz half width is given in Fig. 9.

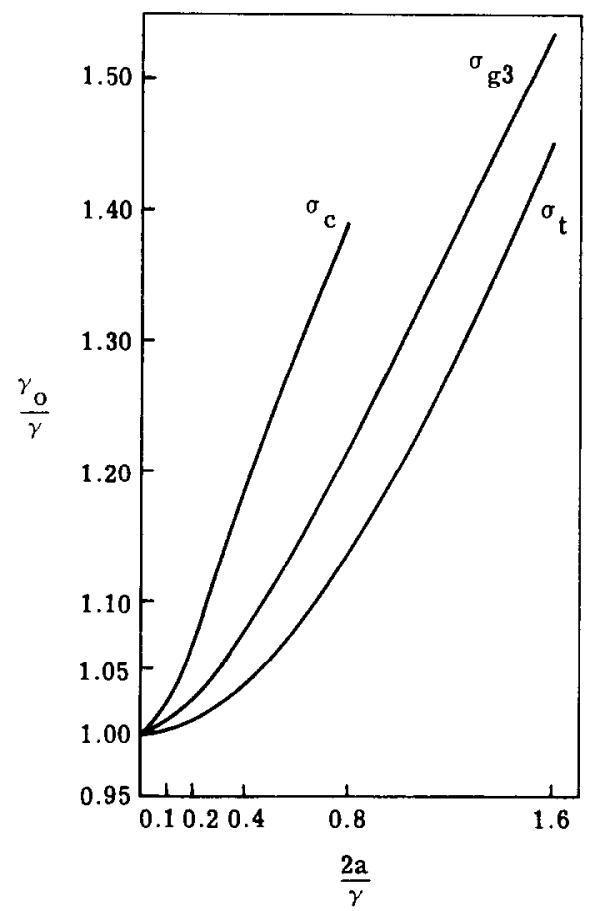

FIG. 9. A comparison of the effect of the form of $\sigma\left(v, v^{\prime}\right)$ on an expected value of $\gamma_{0} / \gamma$.

\section{CONCLUSIONS}

The procedure developed in Section 3 for the determination of spectral line parameters from observed line profiles has as its goal the elimination of systematic errors arising from an experimental determination of the base line. In this regard, it has succeeded admirably. In addition, it has been found to facilitate greatly the accumulation of data, since the method does not require an evacuation of the absorption cell after a measurement. This is not an insignificant consideration, particularly when the gas is highly reactive or polymeric (e.g. HF). For this reason also, contributions from the distant lines are automatically rejected, 
as long as they are weak and vary slowly across the region over which measurements are made. This has been accomplished without the loss of any of the attractive features associated with the usual direct measurement method. Whereas the direct measurement procedure is not intended to achieve detailed line profile information, it has bcen possible in our laboratory to detect nonLorentzian profiles in collision-broadened lines with the use of the new method.

The development of this more precise, though admittedly more conceptually complicated, procedure is in keeping with the premise that resolution is indeed important in the determination of spectral line parameters. The procedure accounts for the fact that the investigator is limited primarily by the spectral resolution available to him; given this limitation, the procedure minimizes the next source of error, the determination of the absolute 100 per cent transmittance associated with an observed line profile.

\section{REFERENCES}

I. J. R. Nielsen, V. Thornton and E. B. Dale, Rev. Mod. Phys. 16, 307 (1944).

2. C. R. Deprima and S. S. Penner, J. chem. Phys. 22, 757 (1955).

3. H. J. Kostkowski and A. M. BASS, J. Opt. Soc. Am. 44, 1060 (1956).

4. R. E. Meredith and N. F. Kent, Willow Run Laboratories, Report No. 8416-26-T, The University of Michigan 1968

5. G. R. IzATt, Report No. NONr 248(01), Johns Hopkins University, Baltimore (1960).

6. W. F. Herget, W. E. Deeds, N. M. Gailar, R. J. Lovell and A. H. Niflsen, J. Opt. Soc. Am. 52, 113 (1962).

7. T. G. Kyle and J. O. Green, J. Opt. Soc. Am. 55, 895 (1965).

8. J. M. Render and R. J. Lovell. J. Opt. Soc. Am. 56, 51 (1966).

APPENDIX I-INSTRUMENTAL CORRECTION FACTORS FOR LORENTZ LINES EXPECTED FROM A SPECTROMETER HAVING A GAUSS SLIT FUNCTION, $\sigma_{g}\left(v, v^{\prime}\right)$

\begin{tabular}{|c|c|c|c|c|c|c|}
\hline \multirow[b]{2}{*}{$\frac{2 a}{\gamma}$} & \multicolumn{5}{|c|}{$\alpha^{P}=30^{\%}$} & \multirow[b]{2}{*}{$\alpha_{0}^{\prime P}$} \\
\hline & $\alpha_{0}(G)$ & $G$ & $S_{c}$ & $K_{i}^{P}$ & $i_{c}$ & \\
\hline \multirow[t]{8}{*}{0.1} & 0.0 & $x$ & 1.0000 & 1.0018 & 1.0026 & 0.2996 \\
\hline & 0.1 & 18.85 & 1.0724 & 1.0046 & 0.9997 & 0.2989 \\
\hline & 0.3 & 10.85 & 1.1322 & 1.0103 & 0.9943 & 0.2974 \\
\hline & 0.5 & 8.38 & 1.1774 & 1.0161 & 0.9888 & 0.2960 \\
\hline & 0.7 & 7.06 & 1.2168 & 1.0220 & 0.9833 & 0.2946 \\
\hline & 0.9 & 6.20 & 1.2530 & 1.2079 & 0.9777 & 0.2932 \\
\hline & 1.1 & 5.59 & 1.2872 & 1.0339 & 0.9722 & 0.2918 \\
\hline & 1.3 & 5.12 & 1.3200 & 1.0400 & 0.9667 & 0.2903 \\
\hline \multirow[t]{8}{*}{0.2} & 0.0 & $x$ & 1.0000 & 1.0071 & 1.0103 & 0.2982 \\
\hline & 0.1 & 18.86 & 1.0726 & 1.0100 & 1.0074 & 0.2975 \\
\hline & 0.3 & 10.85 & 1.1325 & 1.0157 & 1.0016 & 0.2961 \\
\hline & 0.5 & 8.38 & 1.1777 & 1.0216 & 0.9960 & 0.2947 \\
\hline & 0.7 & 7.06 & 1.2172 & 1.0275 & 0.9905 & 0.2933 \\
\hline & 0.9 & 6.20 & 1.2534 & 1.0335 & 0.9850 & 0.2919 \\
\hline & 1.1 & 5.59 & 1.2876 & 1.0396 & 0.9795 & 0.2904 \\
\hline & 1.3 & 5.13 & 1.3205 & 1.0458 & 0.9739 & 0.2890 \\
\hline
\end{tabular}


APPENDIX I (Continued)

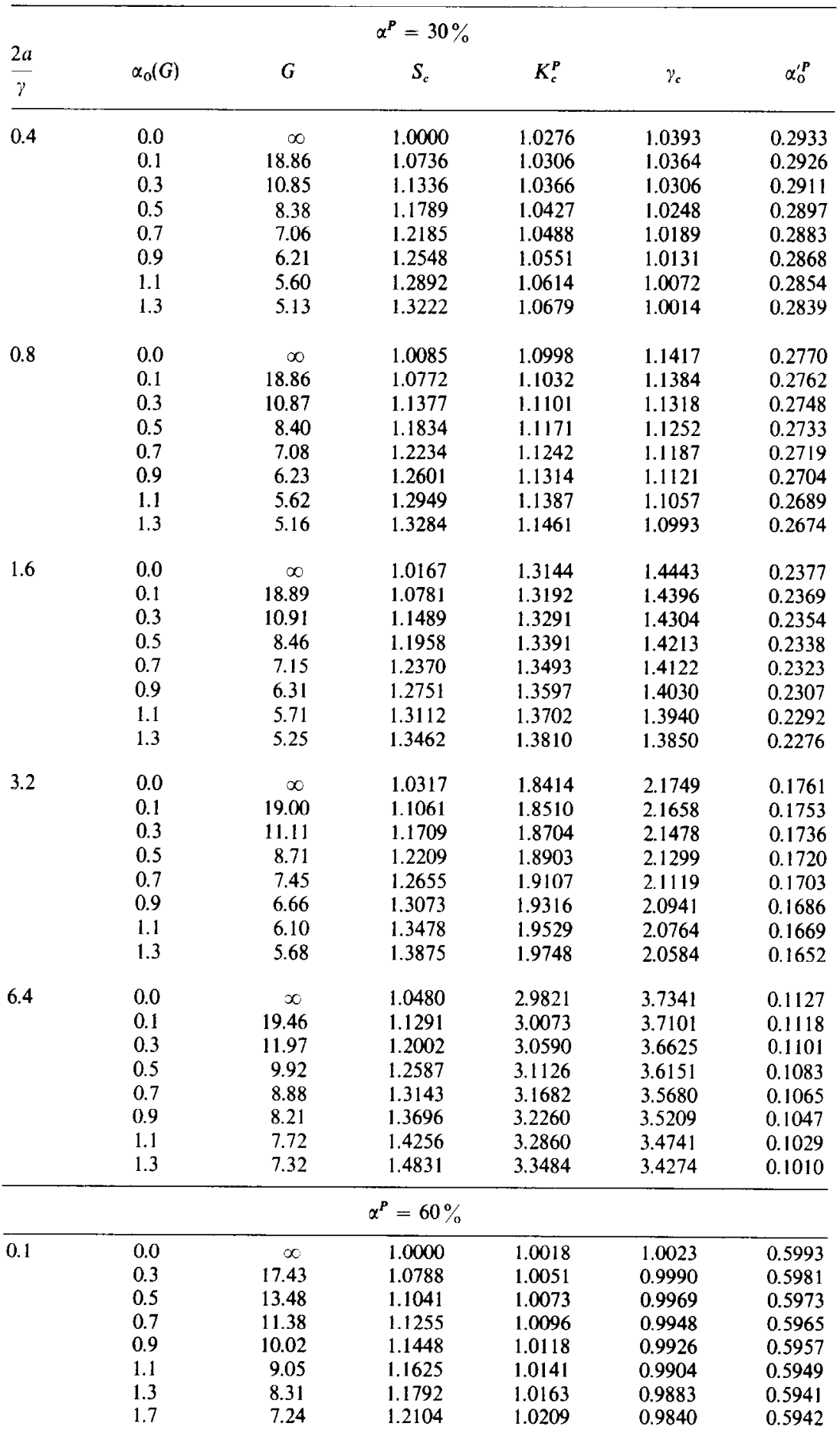


APPENDIX I (Continued)

\begin{tabular}{|c|c|c|c|c|c|c|}
\hline & & & $P=60^{\circ}$ & & & \\
\hline$\frac{2 a}{\gamma}$ & $\alpha_{0}(G)$ & $G$ & $S_{c}$ & $K_{c}^{P}$ & $\gamma_{c}$ & $\alpha_{0}^{\prime P}$ \\
\hline 0.2 & 0.0 & $\infty$ & 1.0005 & 1.0072 & 1.0093 & 0.5974 \\
\hline & 0.3 & 17.44 & 1.0795 & 1.0105 & 1.0059 & 0.5962 \\
\hline & 0.5 & 13.48 & 1.1048 & 1.0127 & 1.0036 & 0.5954 \\
\hline & 0.7 & 11.38 & 1.1263 & 1.0150 & 1.0014 & 0.5945 \\
\hline & 0.9 & 10.02 & 1.1456 & 1.0173 & 0.9992 & 0.5937 \\
\hline & 1.1 & 9.05 & 1.1634 & 1.0196 & 0.9970 & 0.5929 \\
\hline & 1.3 & 8.31 & 1.1801 & 1.0219 & 0.9949 & 0.5921 \\
\hline & 1.7 & 7.24 & 1.2113 & 1.0265 & 0.9905 & 0.5904 \\
\hline 0.4 & 0.0 & $\infty$ & 1.0032 & 1.0280 & 1.0358 & 0.5899 \\
\hline & 0.3 & 17.44 & 1.0822 & 1.0315 & 1.0324 & 0.5887 \\
\hline & 0.5 & 13.49 & 1.1076 & 1.0338 & 1.0301 & 0.5878 \\
\hline & 0.7 & 11.38 & 1.1292 & 1.0362 & 1.0278 & 0.5870 \\
\hline & 0.9 & 10.02 & 1.1486 & 1.0385 & 1.0255 & 0.5862 \\
\hline & 1.1 & 9.05 & 1.1665 & 1.0409 & 1.0232 & 0.5853 \\
\hline & 1.3 & 8.31 & 1.1833 & 1.0433 & 1.0209 & 0.5845 \\
\hline & 1.7 & 7.25 & 1.2147 & 1.0482 & 1.0163 & 0.5828 \\
\hline 0.8 & 0.0 & $\infty$ & 1.0142 & 1.1036 & 1.1311 & 0.5641 \\
\hline & 0.3 & 17.44 & 1.0918 & 1.1076 & 1.1272 & 0.5627 \\
\hline & 0.5 & 13.50 & 1.1177 & 1.1103 & 1.1246 & 0.5619 \\
\hline & 0.7 & 11.39 & 1.1397 & 1.1130 & 1.1219 & 0.5610 \\
\hline & 0.9 & 10.03 & 1.1595 & 1.1158 & 1.1193 & 0.5601 \\
\hline & 1.1 & 9.07 & 1.1777 & 1.1185 & 1.1167 & 0.5592 \\
\hline & 1.3 & 8.33 & 1.1949 & 1.1213 & 1.1141 & 0.5583 \\
\hline & 1.5 & 7.26 & 1.2270 & 1.1269 & 1.1090 & 0.5565 \\
\hline 1.6 & 0.0 & $\infty$ & 1.0386 & 1.3374 & 1.4225 & 0.4960 \\
\hline & 0.3 & 17.47 & 1.1182 & 1.3433 & 1.4169 & 0.4944 \\
\hline & 0.5 & 13.53 & 1.1455 & 1.3473 & 1.4131 & 0.4934 \\
\hline & 0.7 & 11.44 & 1.1687 & 1.3513 & 1.4094 & 0.4924 \\
\hline & 0.9 & 10.09 & 1.1896 & 1.3553 & 1.4057 & 0.4914 \\
\hline & 1.1 & 9.12 & 1.2089 & 1.3594 & 1.4019 & 0.4904 \\
\hline & 1.3 & 8.39 & 1.2271 & 1.3635 & 1.3983 & 0.4893 \\
\hline & 1.7 & 7.33 & 1.2613 & 1.3718 & 1.3909 & 0.4872 \\
\hline 3.2 & 0.0 & $\infty$ & 1.0794 & 1.9263 & 2.1439 & 0.3785 \\
\hline & 0.3 & 17.59 & 1.1676 & 1.9385 & 2.1326 & 0.3767 \\
\hline & 0.5 & 13.69 & 1.1976 & 1.9468 & 2.1251 & 0.3754 \\
\hline & 0.7 & 11.62 & 1.2234 & 1.9552 & 2.1176 & 0.3742 \\
\hline & 0.9 & 10.29 & 1.2467 & 1.9636 & 2.1102 & 0.3729 \\
\hline & 1.1 & 9.35 & 1.2684 & 1.9721 & 2.1027 & 0.3716 \\
\hline & 1.3 & 8.64 & 1.2891 & 1.9808 & 2.0953 & 0.3704 \\
\hline & 1.7 & 7.63 & 1.3283 & 1.9983 & 2.0803 & 0.3678 \\
\hline 6.4 & 0.0 & $\infty$ & 1.1260 & 3.2104 & 3.6987 & 0.2483 \\
\hline & 0.3 & 18.09 & 1.2267 & 3.2446 & 3.6682 & 0.2460 \\
\hline & 0.5 & 14.35 & 1.2612 & 3.2678 & 3.6479 & 0.2445 \\
\hline & 0.7 & 12.43 & 1.2915 & 3.2915 & 3.6278 & 0.2430 \\
\hline & 0.9 & 11.25 & 1.3197 & 3.3155 & 3.6077 & 0.2415 \\
\hline & 1.1 & 10.43 & 1.3467 & 3.3399 & 3.5874 & 0.2399 \\
\hline & 1.3 & 9.83 & 1.3730 & 3.3647 & 3.5673 & 0.2384 \\
\hline & 1.7 & 8.98 & 1.4248 & 3.4156 & 3.5271 & 0.2353 \\
\hline
\end{tabular}


APPENDIX I (Continued)

\begin{tabular}{|c|c|c|c|c|c|c|}
\hline & & & $\boldsymbol{P}=99 \%$ & & & \\
\hline$\frac{2 a}{\gamma}$ & $\alpha_{0}(G)$ & $G$ & $S_{c}$ & $K_{c}^{P}$ & $\gamma_{c}$ & $\alpha_{0}^{\prime P}$ \\
\hline 0.1 & 0.0 & $\infty$ & 1.0010 & 1.0018 & 1.0007 & 0.9899 \\
\hline & 0.3 & 39.14 & 1.0347 & 1.0025 & 1.0000 & 0.9899 \\
\hline & 0.7 & 25.58 & 1.0534 & 1.0033 & 0.9992 & 0.9898 \\
\hline & 1.1 & 20.38 & 1.0677 & 1.0042 & 0.9983 & 0.9898 \\
\hline & 1.5 & 17.43 & 1.0798 & 1.0051 & 0.9974 & 0.9898 \\
\hline & 1.9 & 15.46 & 1.0907 & 1.0060 & 0.9966 & 0.9897 \\
\hline & 2.3 & 14.03 & 1.1007 & 1.0069 & 0.9957 & 0.9897 \\
\hline 0.2 & 0.0 & $\infty$ & 1.0041 & 1.0073 & 1.0028 & 0.9897 \\
\hline & 0.3 & 39.14 & 1.0380 & 1.0080 & 1.0021 & 0.9896 \\
\hline & 0.7 & 25.59 & 1.0569 & 1.0089 & 1.0012 & 0.9896 \\
\hline & 1.1 & 20.38 & 1.0712 & 1.0098 & 1.0003 & 0.9895 \\
\hline & 1.5 & 17.43 & 1.0834 & 1.0107 & 0.9994 & 0.9895 \\
\hline & 1.9 & 15.46 & 1.0943 & 1.0116 & 0.9985 & 0.9895 \\
\hline & 2.3 & 14.03 & 1.1044 & 1.0125 & 0.9977 & 0.9894 \\
\hline 0.4 & 0.0 & $\infty$ & 1.0162 & 1.0309 & 1.0132 & 0.9885 \\
\hline & 0.3 & 39.14 & 1.0508 & 1.0315 & 1.0125 & 0.9885 \\
\hline & 0.7 & 25.59 & 1.0701 & 1.0325 & 1.0115 & 0.9884 \\
\hline & 1.1 & 20.38 & 1.0848 & 1.0334 & 1.0105 & 0.9884 \\
\hline & 1.5 & 17.43 & 1.0974 & 1.0344 & 1.0096 & 0.9883 \\
\hline & 1.9 & 15.46 & 1.1086 & 1.0353 & 1.0086 & 0.9883 \\
\hline & 2.3 & 14.04 & 1.1189 & 1.0362 & 1.0076 & 0.9883 \\
\hline 0.8 & 0.0 & $\infty$ & 1.0592 & 1.1366 & 1.0751 & 0.9826 \\
\hline & 0.3 & 39.14 & 1.0968 & 1.1375 & 1.0742 & 0.9826 \\
\hline & 0.7 & 25.59 & 1.1178 & 1.1386 & 1.0731 & 0.9825 \\
\hline & 1.1 & 20.39 & 1.1339 & 1.1398 & 1.0719 & 0.9824 \\
\hline & 1.5 & 17.44 & 1.1476 & 1.1409 & 1.0708 & 0.9823 \\
\hline & 1.9 & 15.45 & 1.1599 & 1.1421 & 1.0696 & 0.9823 \\
\hline & 2.3 & 14.04 & 1.1712 & 1.1432 & 1.0685 & 0.9822 \\
\hline 1.6 & 0.0 & $\infty$ & 1.1869 & 1.5371 & 1.3592 & 0.9500 \\
\hline & 0.3 & 39.16 & 1.2195 & 1.5387 & 1.3577 & 0.9499 \\
\hline & 0.7 & 25.61 & 1.2456 & 1.5408 & 1.3558 & 0.9497 \\
\hline & 1.1 & 20.41 & 1.2656 & 1.5428 & 1.3539 & 0.9495 \\
\hline & 1.5 & 17.47 & 1.2827 & 1.5449 & 1.3519 & 0.9492 \\
\hline & 1.9 & 15.51 & 1.2981 & 1.5470 & 1.3500 & 0.9490 \\
\hline & 2.3 & 14.08 & 1.3123 & 1.5492 & 1.3480 & 0.9488 \\
\hline 3.2 & 0.0 & $\infty$ & 1.3584 & 2.1507 & 2.1096 & 0.8344 \\
\hline & 0.3 & 39.21 & 1.4204 & 2.5150 & 2.1063 & 0.8339 \\
\hline & 0.7 & 25.69 & 1.4561 & 2.5707 & 2.1017 & 0.8333 \\
\hline & 1.1 & 20.52 & 1.4836 & 2.5765 & 2.0973 & 0.8326 \\
\hline & 1.5 & 17.58 & 1.5074 & 2.5824 & 2.0928 & 0.8319 \\
\hline & 1.9 & 15.64 & 1.5289 & 2.5883 & 2.0883 & 0.8312 \\
\hline & 2.3 & 14.23 & 1.5488 & 2.5943 & 2.0837 & 0.8305 \\
\hline 6.4 & 0.0 & $\infty$ & 1.5419 & 4.7244 & 3.6898 & 0.6227 \\
\hline & 0.3 & 39.42 & 1.6474 & 4.7390 & 3.6799 & 0.6216 \\
\hline & 0.7 & 26.02 & 1.6960 & 4.7586 & 3.6669 & 0.6201 \\
\hline & 1.1 & 20.93 & 1.7341 & 4.7786 & 3.6539 & 0.6185 \\
\hline & 1.5 & 18.07 & 1.7674 & 4.7988 & 3.6410 & 0.6170 \\
\hline & 1.9 & 16.19 & 1.7979 & 4.8192 & 3.6279 & 0.6154 \\
\hline & 2.3 & 14.84 & 1.8267 & 4.8399 & 3.6149 & 0.6138 \\
\hline
\end{tabular}


APPENDIX II - INSTRUMENTAL CORRECTION FACTORS FOR DOPPLER LINES EXPECTEI FROM A SPECTROMETER HAVING A GAUSS SLIT FUNCTION

\begin{tabular}{|c|c|c|c|c|c|c|}
\hline & & & $\alpha^{P}=30$ & & & \\
\hline$\frac{2 a}{?}$ & $\alpha_{0}(G)$ & $G$ & $S_{c}$ & $K_{c}^{P}$ & $\gamma_{c}$ & $x_{0}^{\prime P}$ \\
\hline 0.1 & 0.0 & $\infty$ & 1.0001 & 1.0012 & 1.0010 & 0.2997 \\
\hline & 0.1 & 2.91 & 1.0085 & 1.0041 & 0.9990 & 0.2990 \\
\hline & 0.3 & 2.63 & 1.0235 & 1.0098 & 0.9950 & 0.2976 \\
\hline & 0.5 & 2.48 & 1.0378 & 1.0155 & 0.9909 & 0.2962 \\
\hline & 0.7 & 2.38 & 1.0519 & 1.0214 & 0.9869 & 0.2948 \\
\hline & 0.9 & 2.31 & 1.0658 & 1.0273 & 0.9828 & 0.2933 \\
\hline & 1.1 & 2.04 & 1.0797 & 1.0333 & 0.9788 & 0.2919 \\
\hline 0.2 & 0.0 & $x$ & 1.0006 & 1.0050 & 1.0041 & 0.2988 \\
\hline & 0.1 & 2.92 & 1.0090 & 1.0078 & 1.0021 & 0.2981 \\
\hline & 0.3 & 2.64 & 1.0241 & 1.0136 & 0.9980 & 0.2966 \\
\hline & 0.5 & 2.49 & 1.0385 & 1.0194 & 0.9939 & 0.2952 \\
\hline & 0.7 & 2.39 & 1.0526 & 1.0253 & 0.9899 & 0.2938 \\
\hline & 0.9 & 2.31 & 1.0666 & 1.0313 & 0.9858 & 0.2924 \\
\hline & 1.1 & 2.25 & 1.0806 & 1.0373 & 0.9817 & 0.2910 \\
\hline 0.4 & 0.0 & $\infty$ & 1.0024 & 1.0199 & 1.0166 & 0.2951 \\
\hline & 0.1 & 2.96 & 1.0109 & 1.0229 & 1.0144 & 0.2944 \\
\hline & 0.3 & 2.67 & 1.0262 & 1.0288 & 1.0102 & 0.2930 \\
\hline & 0.5 & 2.52 & 1.0409 & 1.0348 & 1.0060 & 0.2916 \\
\hline & 0.7 & 2.42 & 1.0552 & 1.0408 & 1.0017 & 0.2901 \\
\hline & 0.9 & 2.34 & 1.0695 & 1.0470 & 0.9975 & 0.2887 \\
\hline & 1.1 & 2.28 & 1.0837 & 1.0532 & 0.9933 & 0.2873 \\
\hline 0.8 & 0.0 & $\infty$ & 1.0089 & 1.0790 & 1.0660 & 0.2815 \\
\hline & 0.1 & 3.12 & 1.0180 & 1.0823 & 1.0636 & 0.2808 \\
\hline & 0.3 & 2.80 & 1.0343 & 1.0889 & 1.0589 & 0.2793 \\
\hline & 0.5 & 2.65 & 1.0500 & 1.0956 & 1.0542 & 0.2779 \\
\hline & 0.7 & 2.54 & 1.0653 & 1.1024 & 1.0495 & 0.2764 \\
\hline & 0.9 & 2.45 & 1.0806 & 1.1093 & 1.0448 & 0.2750 \\
\hline & 1.1 & 2.38 & 1.0958 & 1.1163 & 1.0401 & 0.2735 \\
\hline 1.6 & 0.0 & $\infty$ & 1.0276 & 1.2964 & 1.2534 & 0.2405 \\
\hline & 0.1 & 3.64 & 1.0387 & 1.3012 & 1.2500 & 0.2398 \\
\hline & 0.3 & 3.27 & 1.0586 & 1.3108 & 1.2433 & 0.2382 \\
\hline & 0.5 & 3.07 & 1.0777 & 1.3205 & 1.2366 & 0.2367 \\
\hline & 0.7 & 2.94 & 1.0965 & 1.3304 & 1.2298 & 0.2352 \\
\hline & 0.9 & 2.84 & 1.1153 & 1.3405 & 1.2231 & 0.2336 \\
\hline & 1.1 & 2.75 & 1.1341 & 1.3508 & 1.2164 & 0.2321 \\
\hline 3.2 & 0.0 & $\infty$ & 1.0605 & 1.9680 & 1.8442 & 0.1658 \\
\hline & 0.1 & 5.16 & 1.0774 & 1.9790 & 1.8367 & 0.1649 \\
\hline & 0.3 & 4.57 & 1.1078 & 2.0013 & 1.8218 & 0.1632 \\
\hline & 0.5 & 4.27 & 1.1372 & 2.0241 & 1.8069 & 0.1616 \\
\hline & 0.7 & 4.06 & 1.1665 & 2.0474 & 1.7920 & 0.1599 \\
\hline & 0.9 & 3.90 & 1.1960 & 2.0714 & 1.7771 & 0.1582 \\
\hline & 1.1 & 3.76 & 1.2259 & 2.0960 & 1.7622 & 0.1565 \\
\hline 6.4 & 0.0 & $\infty$ & 1.0910 & 3.6150 & 3.3014 & 0.0940 \\
\hline & 0.1 & 8.60 & 1.1213 & 3.6521 & 3.2769 & 0.0930 \\
\hline & 0.3 & 7.49 & 1.1768 & 3.7286 & 3.2283 & 0.0912 \\
\hline & 0.5 & 6.91 & 1.2319 & 3.8085 & 3.1798 & 0.0894 \\
\hline & 0.7 & 6.50 & 1.2882 & 3.8921 & 3.1315 & 0.0876 \\
\hline & 0.9 & 6.18 & 1.3466 & 3.9797 & 3.0831 & 0.0857 \\
\hline & 1.1 & 5.91 & 1.4076 & 4.0715 & 3.0350 & 0.0839 \\
\hline
\end{tabular}


APPENDIX II (Continued)

\begin{tabular}{|c|c|c|c|c|c|c|}
\hline & & & $\alpha^{P}=60^{\circ}$ & & & \\
\hline$\frac{2 a}{\gamma}$ & $x_{0}(G)$ & $G$ & $S_{c}$ & $K_{c}^{P}$ & $\gamma_{c}$ & $\alpha_{0}^{\prime P}$ \\
\hline 0.1 & 0.0 & $\infty$ & 1.0004 & 1.0013 & 1.0007 & 0.5995 \\
\hline & 0.1 & 3.14 & 1.0038 & 1.0023 & 0.9999 & 0.5991 \\
\hline & 0.3 & 2.88 & 1.0101 & 1.0045 & 0.9983 & 0.5983 \\
\hline & 0.5 & 2.74 & 1.0160 & 1.0068 & 0.9968 & 0.5975 \\
\hline & 0.7 & 2.65 & 1.0218 & 1.0090 & 0.9952 & 0.5967 \\
\hline & 0.9 & 2.58 & 1.0275 & 1.0112 & 0.9936 & 0.5959 \\
\hline & 1.1 & 2.53 & 1.0331 & 1.0135 & 0.9920 & 0.5951 \\
\hline 0.2 & 0.0 & $\infty$ & 1.0016 & 1.0050 & 1.0028 & 0.5982 \\
\hline & 0.1 & 3.15 & 1.0051 & 1.0061 & 1.0020 & 0.5978 \\
\hline & 0.3 & 2.89 & 1.0113 & 1.0083 & 1.0003 & 0.5970 \\
\hline & 0.5 & 2.75 & 1.0173 & 1.0106 & 0.9988 & 0.5962 \\
\hline & 0.7 & 2.66 & 1.0231 & 1.0128 & 0.9972 & 0.5953 \\
\hline & 0.9 & 2.59 & 1.0288 & 1.0151 & 0.9956 & 0.5945 \\
\hline & 1.1 & 2.54 & 1.0345 & 1.0174 & 0.9940 & 0.5937 \\
\hline 0.4 & 0.0 & $\infty$ & 1.0062 & 1.0201 & 1.0113 & 0.5927 \\
\hline & 0.1 & 3.19 & 1.0098 & 1.0213 & 1.0105 & 0.5923 \\
\hline & 0.3 & 2.92 & 1.0162 & 1.0236 & 1.0088 & 0.5915 \\
\hline & 0.5 & 2.79 & 1.0223 & 1.0259 & 1.0072 & 0.5906 \\
\hline & 0.7 & 2.70 & 1.0283 & 1.0282 & 1.0055 & 0.5898 \\
\hline & 0.9 & 2.63 & 1.0341 & 1.0305 & 1.0038 & 0.5890 \\
\hline & 1.1 & 2.57 & 1.0399 & 1.0329 & 1.0022 & 0.5882 \\
\hline 0.8 & 0.0 & $\infty$ & 1.0235 & 1.0819 & 1.0487 & 0.5713 \\
\hline & 0.1 & 3.36 & 1.0274 & 1.0832 & 1.0478 & 0.5708 \\
\hline & 0.3 & 3.07 & 1.0343 & 1.0858 & 1.0459 & 0.5700 \\
\hline & 0.5 & 2.93 & 1.0410 & 1.0884 & 1.0441 & 0.5691 \\
\hline & 0.7 & 2.83 & 1.0474 & 1.0910 & 1.0422 & 0.5682 \\
\hline & 0.9 & 2.75 & 1.0538 & 1.0936 & 1.0404 & 0.5674 \\
\hline & 1.1 & 2.69 & 1.0601 & 1.0963 & 1.0385 & 0.5665 \\
\hline 1.6 & 0.0 & $x$ & 1.0741 & 1.3245 & 1.2137 & 0.4993 \\
\hline & 0.1 & 3.94 & 1.0792 & 1.3265 & 1.2124 & 0.4988 \\
\hline & 0.3 & 3.59 & 1.0882 & 1.3303 & 1.2097 & 0.4978 \\
\hline & 0.5 & 3.41 & 1.0968 & 1.3342 & 1.2070 & 0.4968 \\
\hline & 0.7 & 3.29 & 1.1051 & 1.3381 & 1.2043 & 0.4958 \\
\hline & 0.9 & 3.20 & 1.1134 & 1.3421 & 1.2016 & 0.4948 \\
\hline & 1.1 & 3.12 & 1.1216 & 1.3461 & 1.1989 & 0.4937 \\
\hline 3.2 & 0.0 & $\infty$ & 1.1635 & 2.1113 & 1.7887 & 0.3521 \\
\hline & 0.1 & 5.58 & 1.1719 & 2.1162 & 1.7856 & 0.3514 \\
\hline & 0.3 & 5.04 & 1.1870 & 2.1261 & 1.7794 & 0.3501 \\
\hline & 0.5 & 4.76 & 1.2013 & 2.1360 & 1.7731 & 0.3488 \\
\hline & 0.7 & 4.57 & 1.2154 & 2.1461 & 1.7669 & 0.3475 \\
\hline & 0.9 & 4.43 & 1.2293 & 2.1563 & 1.7606 & 0.3462 \\
\hline & 1.1 & 4.31 & 1.2431 & 2.1666 & 1.7544 & 0.3449 \\
\hline 6.4 & 0.0 & $\infty$ & 1.2452 & 4.0673 & 3.2402 & 0.2017 \\
\hline & 0.1 & 9.33 & 1.2612 & 4.0854 & 3.2295 & 0.2009 \\
\hline & 0.3 & 8.31 & 1.2905 & 4.1222 & 3.2083 & 0.1993 \\
\hline & 0.5 & 7.79 & 1.3187 & 4.1598 & 3.1870 & 0.1977 \\
\hline & 0.7 & 7.43 & 1.3465 & 4.1982 & 3.1658 & 0.1961 \\
\hline & 0.9 & 7.15 & 1.3744 & 4.2373 & 3.1445 & 0.1945 \\
\hline & 1.1 & 6.91 & 1.4025 & 4.2773 & 3.1233 & 0.1928 \\
\hline
\end{tabular}


APPENDIX II (Continued)

\begin{tabular}{|c|c|c|c|c|c|c|}
\hline \multicolumn{7}{|c|}{$\alpha^{P}=99 \%$} \\
\hline$\frac{2 a}{\gamma}$ & $\alpha_{0}(G)$ & $G$ & $S_{c}$ & $K_{c}^{P}$ & $\gamma_{c}$ & $\alpha_{0}^{\prime P}$ \\
\hline \multirow[t]{6}{*}{0.1} & 0.0 & $\infty$ & 1.0020 & 1.0013 & 0.9984 & 0.9899 \\
\hline & 0.3 & 3.26 & 1.0042 & 1.0019 & 0.9979 & 0.9899 \\
\hline & 0.7 & 3.06 & 1.0068 & 1.0028 & 0.9973 & 0.9899 \\
\hline & 1.1 & 2.95 & 1.0093 & 1.0037 & 0.9967 & 0.9898 \\
\hline & 1.5 & 2.88 & 1.0117 & 1.0046 & 0.9960 & 0.9898 \\
\hline & 1.9 & 2.81 & 1.0142 & 1.0055 & 0.9954 & 0.9897 \\
\hline \multirow[t]{6}{*}{0.2} & 0.0 & $\infty$ & 1.0081 & 1.0051 & 0.9937 & 0.9898 \\
\hline & 0.3 & 3.27 & 1.0103 & 1.0058 & 0.9933 & 0.9897 \\
\hline & 0.7 & 3.07 & 1.0130 & 1.0066 & 0.9926 & 0.9897 \\
\hline & 1.1 & 2.96 & 1.0155 & 1.0075 & 0.9920 & 0.9897 \\
\hline & 1.5 & 2.89 & 1.0180 & 1.0084 & 0.9914 & 0.9896 \\
\hline & 1.9 & 2.82 & 1.0205 & 1.0093 & 0.9907 & 0.9896 \\
\hline \multirow[t]{6}{*}{0.4} & 0.0 & $\infty$ & 1.0325 & 1.0217 & 0.9773 & 0.9890 \\
\hline & 0.3 & 3.31 & 1.0348 & 1.0224 & 0.9768 & 0.9889 \\
\hline & 0.7 & 3.11 & 1.0376 & 1.0233 & 0.9761 & 0.9889 \\
\hline & 1.1 & 3.00 & 1.0404 & 1.0242 & 0.9755 & 0.9888 \\
\hline & 1.5 & 3.92 & 1.0430 & 1.0251 & 0.9748 & 0.9888 \\
\hline & 1.9 & 3.86 & 1.0456 & 1.0261 & 0.9741 & 0.9888 \\
\hline \multirow[t]{6}{*}{0.8} & 0.0 & $\infty$ & 1.1294 & 1.1091 & 0.9437 & 0.9843 \\
\hline & 0.3 & 3.48 & 1.1323 & 1.1099 & 0.9431 & 0.9842 \\
\hline & 0.7 & 3.27 & 1.1358 & 1.1110 & 0.9423 & 0.9842 \\
\hline & 1.1 & 3.15 & 1.1393 & 1.1121 & 0.9415 & 0.984 \\
\hline & 1.5 & 3.07 & 1.0426 & 1.1132 & 0.9407 & 0.9840 \\
\hline & 1.9 & 3.00 & 1.1459 & 1.1143 & 0.9400 & 0.9840 \\
\hline \multirow[t]{7}{*}{1.6} & 0.0 & $\infty$ & 1.4454 & 1.6176 & 1.0490 & 0.9420 \\
\hline & 0.3 & 4.07 & 1.4510 & 1.6194 & 1.0479 & 0.9418 \\
\hline & 0.7 & 3.81 & 1.4578 & 1.6216 & 1.0466 & 0.9416 \\
\hline & 1.1 & 3.66 & 1.4643 & 1.6240 & 1.0452 & 0.9413 \\
\hline & 1.5 & 3.55 & 1.4708 & 1.6263 & 1.0438 & 0.9411 \\
\hline & 1.9 & 3.46 & 1.4771 & 1.6286 & 1.0425 & 0.9408 \\
\hline & & $\infty$ & & & & \\
\hline \multirow[t]{6}{*}{3.2} & 0.0 & 5.68 & 1.9795 & 3.3894 & 1.6377 & 0.7430 \\
\hline & 0.3 & 5.25 & 1.9943 & 3.3971 & 1.6347 & 0.7422 \\
\hline & 0.7 & 5.01 & 2.0122 & 3.4072 & 1.6307 & 0.7412 \\
\hline & 1.1 & 4.83 & 2.0295 & 3.4174 & 1.6266 & 0.7401 \\
\hline & 1.5 & 4.69 & 2.0464 & 3.4277 & 1.6226 & 0.7391 \\
\hline & 1.9 & & 2.0631 & 3.4381 & 1.6185 & 0.7380 \\
\hline \multirow[t]{6}{*}{6.4} & 0.0 & $\infty$ & 2.4338 & 7.6971 & 3.0959 & 0.4503 \\
\hline & 0.3 & 9.26 & 2.4703 & 7.7360 & 3.0839 & 0.4486 \\
\hline & 0.7 & 8.44 & 2.5152 & 7.7886 & 3.0679 & 0.4464 \\
\hline & 1.1 & 7.98 & 2.5587 & 7.8421 & 3.0518 & 0.4441 \\
\hline & 1.5 & 7.64 & 2.6016 & 7.8966 & 3.0358 & 0.4419 \\
\hline & 1.9 & 7.37 & 2.6445 & 7.9521 & 3.0197 & 0.4396 \\
\hline
\end{tabular}


APPENDIX III--INSTRUMENTAL CORRECTION FACTORS FOR VOIGT LINES EXPECTED FROM A SPECTROMETER HAVING A GAUSS SLIT FUNCTION $\sigma_{g}\left(v, v^{\prime}\right)$ FOR $\alpha^{p}=30 \%$

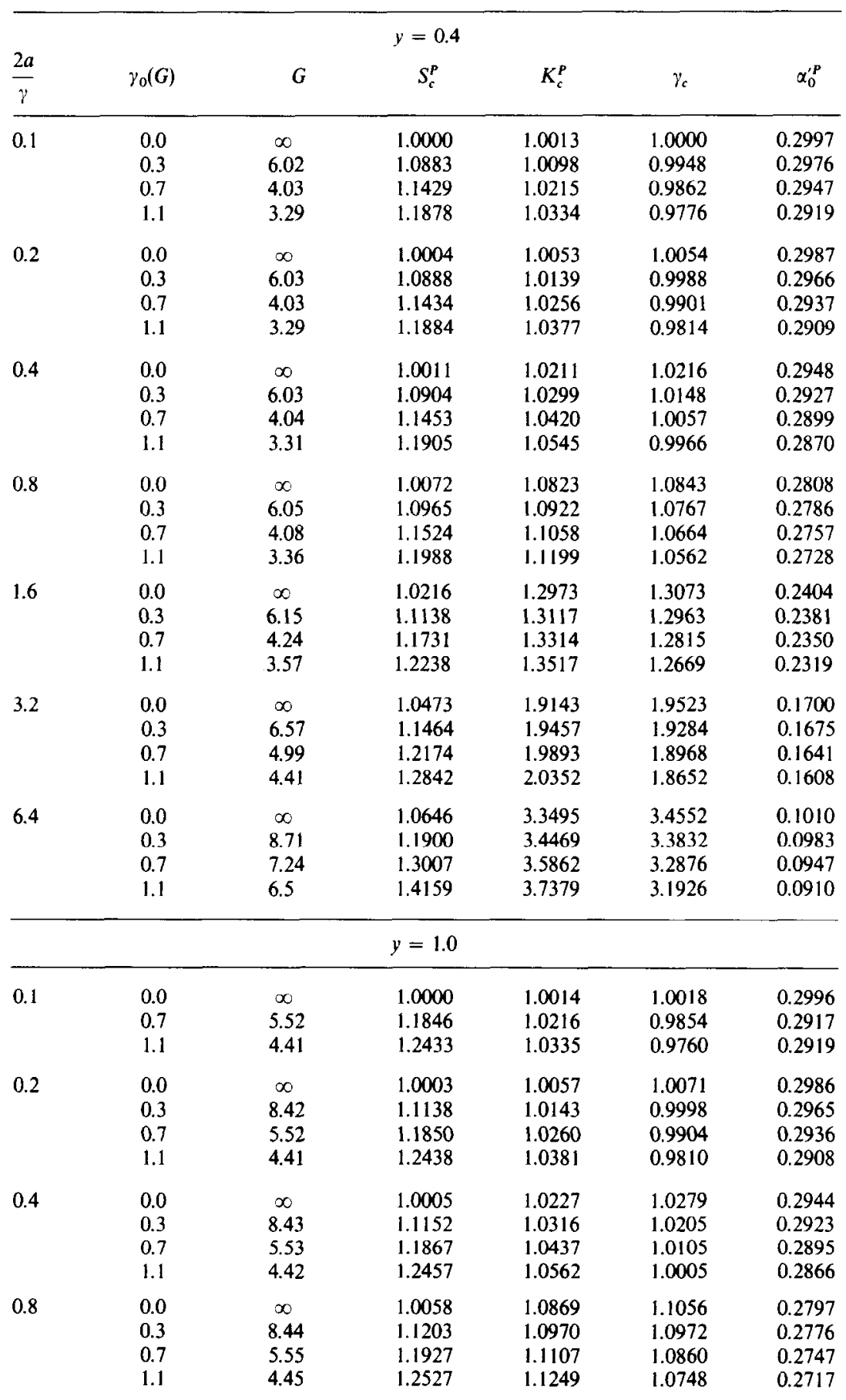


APPENDIX III (Continued)

\begin{tabular}{|c|c|c|c|c|c|c|}
\hline \multirow[b]{2}{*}{$\frac{2 a}{\gamma}$} & \multicolumn{5}{|c|}{$y=1.0$} & \multirow[b]{2}{*}{$\alpha_{0}^{\prime P}$} \\
\hline & $\gamma_{0}(G)$ & $G$ & $S_{c}^{P}$ & $K_{c}^{P}$ & $i_{c}$ & \\
\hline \multirow[t]{4}{*}{1.6} & 0.0 & $x$ & 1.0175 & 1.3006 & 1.3634 & 0.2398 \\
\hline & 0.3 & 8.50 & 1.1346 & 1.3150 & 1.3513 & 0.2376 \\
\hline & 0.7 & 5.65 & 1.2099 & 1.3348 & 1.3352 & 0.2345 \\
\hline & 1.1 & 4.57 & 1.2731 & 1.3553 & 1.3192 & 0.2314 \\
\hline \multirow[t]{4}{*}{3.2} & 0.0 & $x$ & 1.0383 & 1.8736 & 2.0510 & 0.1733 \\
\hline & 0.3 & 8.76 & 1.1617 & 1.9036 & 2.0258 & 0.1709 \\
\hline & 0.7 & 6.08 & 1.2451 & 1.9454 & 1.9924 & 0.1675 \\
\hline & 1.1 & 5.13 & 1.3192 & 1.9891 & 1.9591 & 0.1642 \\
\hline \multirow[t]{4}{*}{6.4} & 0.0 & $\infty$ & 1.0464 & 3.1579 & 3.5841 & 0.1068 \\
\hline & 0.3 & 10.08 & 1.1965 & 3.2442 & 3.5126 & 0.1041 \\
\hline & 0.7 & 7.95 & 1.3080 & 3.3673 & 3.4178 & 0.1005 \\
\hline & 1.1 & 7.04 & 1.4207 & 3.5007 & 3.3237 & 0.0969 \\
\hline
\end{tabular}

APPENDIX IV-INSTRUMENTAL CORRECTION FACTORS FOR LORENTZ LINES EXPF(TTED FROM A SPECTROMETER HAVING A TRIANGULAR SLIT FUNCTION, $\sigma_{\mathrm{f}}\left(v, v^{\prime}\right)$

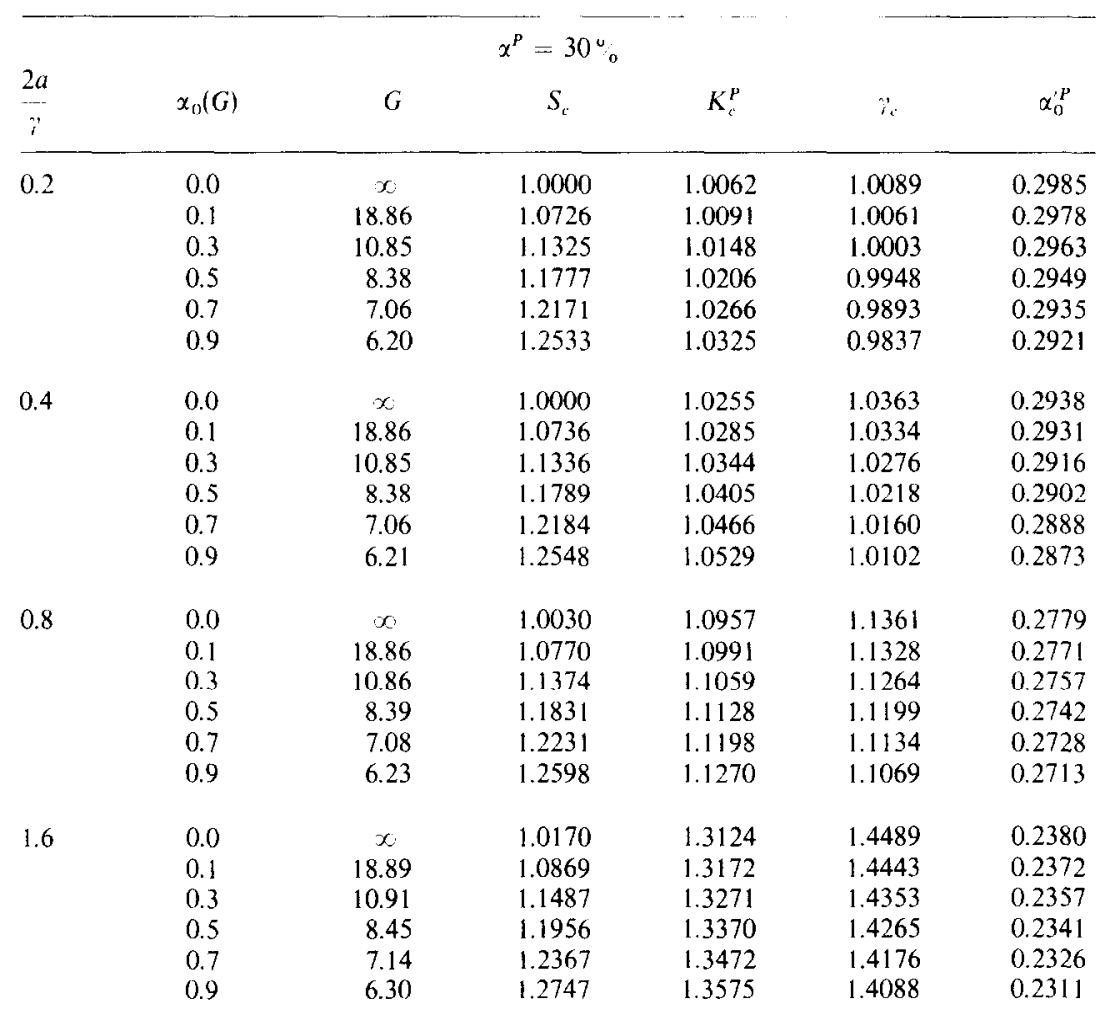


APPENDIX IV (Continued)

\begin{tabular}{|c|c|c|c|c|c|c|}
\hline \multicolumn{7}{|c|}{$\alpha^{P}=30 \%$} \\
\hline$\frac{2 a}{\gamma}$ & $\alpha_{0}(G)$ & $G$ & $S_{\mathrm{c}}$ & $K_{c}^{P}$ & $\gamma_{c}$ & $a_{0}^{\prime P}$ \\
\hline \multirow[t]{6}{*}{3.2} & 0.0 & $\infty$ & 1.0327 & 1.8487 & 2.2364 & 0.1755 \\
\hline & 0.1 & 18.99 & 1.1055 & 1.8583 & 2.2274 & 0.1746 \\
\hline & 0.3 & 11.08 & 1.1702 & 1.8779 & 2.2095 & 0.1730 \\
\hline & 0.5 & 8.68 & 1.2200 & 1.8980 & 2.1917 & 0.1713 \\
\hline & 0.7 & 7.41 & 1.2643 & 1.9185 & 2.1738 & 0.1697 \\
\hline & 0.9 & 6.60 & 1.3057 & 1.9395 & 2.1560 & 0.1680 \\
\hline \multirow[t]{6}{*}{6.4} & 0.0 & $\infty$ & 1.0514 & 2.9900 & 3.9136 & 0.1124 \\
\hline & 0.1 & 19.40 & 1.1288 & 3.0153 & 3.8878 & 0.1116 \\
\hline & 0.3 & 11.79 & 1.1992 & 3.0672 & 3.8364 & 0.1098 \\
\hline & 0.5 & 9.59 & 1.2561 & 3.1211 & 3.7849 & 0.1080 \\
\hline & 0.7 & 8.48 & 1.3092 & 3.1771 & 3.7335 & 0.1062 \\
\hline & 0.9 & 7.81 & 1.3614 & 3.2352 & 3.6819 & 0.1044 \\
\hline \multirow[t]{5}{*}{12.8} & 0.0 & $\infty$ & 1.0663 & 5.2801 & 7.2512 & 0.0653 \\
\hline & 0.3 & 14.68 & 1.2361 & 5.5258 & 6.9828 & 0.0625 \\
\hline & 0.5 & 13.21 & 1.3168 & 5.7033 & 6.8036 & 0.0606 \\
\hline & 0.7 & 12.49 & 1.4019 & 5.8929 & 6.6240 & 0.0587 \\
\hline & 0.9 & 11.97 & 1.4940 & 6.0959 & 6.4440 & 0.0568 \\
\hline \multicolumn{7}{|c|}{$\alpha^{P}=99 \%$} \\
\hline \multirow[t]{7}{*}{0.2} & 0.0 & $\infty$ & 1.0036 & 1.0063 & 1.0023 & 0.9897 \\
\hline & 0.3 & 39.14 & 1.0374 & 1.0070 & 1.0016 & 0.9897 \\
\hline & 0.7 & 25.59 & 1.0563 & 1.0079 & 1.0007 & 0.9896 \\
\hline & 1.1 & 20.38 & 1.0706 & 1.0088 & 0.9999 & 0.9896 \\
\hline & 1.5 & 17.43 & 1.0828 & 1.0097 & 0.9990 & 0.9896 \\
\hline & 1.9 & 15.46 & 1.0937 & 1.0106 & 0.9981 & 0.9895 \\
\hline & 2.3 & 14.03 & 1.1037 & 1.0115 & 0.9972 & 0.9895 \\
\hline \multirow[t]{7}{*}{0.4} & 0.0 & $\infty$ & 1.0143 & 1.0274 & 1.0112 & 0.9887 \\
\hline & 0.3 & 39.14 & 1.0493 & 1.0281 & 1.0105 & 0.9887 \\
\hline & 0.7 & 25.59 & 1.0686 & 1.0290 & 1.0095 & 0.9886 \\
\hline & 1.1 & 20.38 & 1.0832 & 1.0299 & 1.0086 & 0.9886 \\
\hline & 1.5 & 17.43 & 1.0957 & 1.0309 & 1.0076 & 0.9885 \\
\hline & 1.9 & 15.46 & 1.1069 & 1.0318 & 1.0067 & 0.9885 \\
\hline & 2.3 & 14.04 & 1.1172 & 1.0328 & 1.0057 & 0.9884 \\
\hline \multirow[t]{7}{*}{0.8} & 0.0 & $\infty$ & 1.0552 & 1.1205 & 1.0619 & 0.9836 \\
\hline & 0.3 & 39.14 & 1.0921 & 1.1214 & 1.0610 & 0.9835 \\
\hline & 0.7 & 25.59 & 1.1130 & 1.1225 & 1.0599 & 0.9835 \\
\hline & 1.1 & 20.39 & 1.1289 & 1.1236 & 1.5888 & 0.9834 \\
\hline & 1.5 & 17.44 & 1.1425 & 1.1247 & 1.0576 & 0.9833 \\
\hline & 1.9 & 15.47 & 1.1547 & 1.1258 & 1.0565 & 0.9833 \\
\hline & 2.3 & 14.04 & 1.1659 & 1.1269 & 1.0553 & 0.9832 \\
\hline \multirow[t]{7}{*}{1.6} & 0.0 & $\infty$ & 1.1654 & 1.4990 & 1.3275 & 0.9537 \\
\hline & 0.3 & 39.15 & 1.2111 & 1.5004 & 1.3261 & 0.9535 \\
\hline & 0.7 & 25.61 & 1.2369 & 1.5024 & 1.3242 & 0.9534 \\
\hline & 1.1 & 20.41 & 1.2566 & 1.5044 & 1.3224 & 0.9532 \\
\hline & 1.5 & 17.46 & 1.2734 & 1.5064 & 1.3205 & 0.9530 \\
\hline & 1.9 & 15.50 & 1.2886 & 1.5084 & 1.3187 & 0.9528 \\
\hline & 2.3 & 14.08 & 1.3026 & 1.5104 & 1.3168 & 0.9526 \\
\hline
\end{tabular}


APPENDIX IV (Continued)

\begin{tabular}{|c|c|c|c|c|c|c|}
\hline \multicolumn{7}{|c|}{$x^{P}=99 \%$} \\
\hline$\frac{2 a}{\gamma}$ & $a_{0}(G)$ & $G$ & $S_{c}$ & $K_{c}^{P}$ & $\gamma_{c}^{\prime}$ & $a_{0}^{\prime P}$ \\
\hline \multirow{7}{*}{3.2} & 0.0 & $\infty$ & 1.3528 & 2.5448 & 2.1155 & 0.8363 \\
\hline & 0.3 & 39.20 & 1.4143 & 2.5490 & 2.1122 & 0.8358 \\
\hline & 0.7 & 25.68 & 1.4496 & 2.5547 & 2.1078 & 0.8351 \\
\hline & 1.1 & 20.50 & 1.4769 & 2.5604 & 2.1034 & 0.8345 \\
\hline & 1.5 & 17.57 & 1.5004 & 2.5662 & 2.0989 & 0.8338 \\
\hline & 1.9 & 15.62 & 1.5217 & 2.5720 & 2.0945 & 0.8331 \\
\hline & 2.3 & 14.21 & 1.5414 & 2.5779 & 2.0900 & 0.8324 \\
\hline \multirow[t]{7}{*}{6.4} & 0.0 & $\infty$ & 1.5511 & 4.7631 & 3.8119 & 0.6197 \\
\hline & 0.3 & 39.40 & 1.6445 & 4.7780 & 3.8019 & 0.6186 \\
\hline & 0.7 & 25.98 & 1.6929 & 4.7980 & 3.7885 & 0.6170 \\
\hline & 1.1 & 20.88 & 1.7307 & 4.8183 & 3.7751 & 0.6155 \\
\hline & 1.5 & 18.01 & 1.7638 & 4.8388 & 3.7618 & 0.6139 \\
\hline & 1.9 & 16.12 & 1.7941 & 4.8595 & 3.7483 & 0.6124 \\
\hline & 2.3 & 14.75 & 1.8226 & 4.8806 & 3.7349 & 0.6108 \\
\hline \multirow[t]{7}{*}{12.8} & 0.0 & $\infty$ & 1.7237 & 9.1435 & 7.1985 & 0.3957 \\
\hline & 0.3 & 40.18 & 1.8431 & 8.1984 & 7.1626 & 0.3939 \\
\hline & 0.7 & 27.19 & 1.9062 & 8.2729 & 7.1146 & 0.3914 \\
\hline & 1.1 & 22.39 & 1.9573 & 8.3489 & 7.0667 & 0.3890 \\
\hline & 1.5 & 19.78 & 2.0034 & 8.4264 & 7.0184 & 0.3865 \\
\hline & 1.9 & 18.11 & 2.0470 & 8.5056 & 6.9702 & 0.3840 \\
\hline & 2.3 & 16.94 & 2.0892 & 8.5864 & 6.9219 & 0.3815 \\
\hline
\end{tabular}

APPENDIX V-INSTRUMENTAL CORRECTION FACTORS FOR LORENTZ LINES EXPECTED FROM A SPECTROMETER HAVING A CAUCHY SLIT FUNCTION, $\sigma_{c}\left(v, v^{\prime}\right)$

\begin{tabular}{|c|c|c|c|c|c|c|}
\hline \multirow[b]{2}{*}{$\frac{2 a}{\gamma}$} & \multicolumn{4}{|c|}{$\alpha^{P}=30 \%$} & \multirow[b]{2}{*}{$\gamma_{c}$} & \multirow[b]{2}{*}{$x_{0}^{\prime P}$} \\
\hline & $\alpha_{0}(G)$ & $G$ & $S_{c}$ & $K_{c}^{P}$ & & \\
\hline \multirow[t]{8}{*}{0.1} & 0.0 & $\infty$ & 1.0000 & 1.0136 & 1.0195 & 0.2967 \\
\hline & 0.3 & 10.85 & 1.1328 & 1.0223 & 1.0108 & 0.2945 \\
\hline & 0.5 & 8.38 & 1.1781 & 1.0282 & 1.0050 & 0.2931 \\
\hline & 0.7 & 7.06 & 1.2176 & 1.0342 & 0.9993 & 0.2917 \\
\hline & 0.9 & 6.20 & 1.2538 & 1.0403 & 0.9937 & 0.2903 \\
\hline & 1.1 & 5.59 & 1.2881 & 1.0465 & 0.9882 & 0.2888 \\
\hline & 1.3 & 5.13 & 1.3210 & 1.0527 & 0.9826 & 0.2874 \\
\hline & 1.7 & 4.45 & 1.3842 & 1.0655 & 0.9714 & 0.2845 \\
\hline \multirow[t]{8}{*}{0.2} & 0.0 & $\infty$ & 1.0023 & 1.0473 & 1.0667 & 0.2886 \\
\hline & 0.3 & 10.86 & 1.1349 & 1.0567 & 1.0575 & 0.2865 \\
\hline & 0.5 & 8.39 & 1.1804 & 1.0630 & 1.0514 & 0.2851 \\
\hline & 0.7 & 7.07 & 1.2200 & 1.0694 & 1.0454 & 0.2836 \\
\hline & 0.9 & 6.21 & 1.2565 & 1.0759 & 1.0394 & 0.2822 \\
\hline & 1.1 & 5.60 & 1.2910 & 1.0825 & 1.0334 & 0.2807 \\
\hline & 1.3 & 5.14 & 1.3241 & 1.0892 & 1.0273 & 0.2793 \\
\hline & 1.7 & 4.47 & 1.3879 & 1.1029 & 1.0155 & 0.2763 \\
\hline
\end{tabular}


APPENDIX V (Continued)

\begin{tabular}{|c|c|c|c|c|c|c|}
\hline $\begin{array}{c}2 a \\
\gamma\end{array}$ & $\alpha_{0}(G)$ & $G$ & $\begin{array}{c}x^{P}=30 \% \\
S_{c}\end{array}$ & $K_{c}^{P}$ & $\gamma_{c}$ & $\alpha_{0}^{r}$ \\
\hline 0.4 & $\begin{array}{l}0.0 \\
0.3 \\
0.5 \\
0.7 \\
0.9 \\
1.1 \\
1.3 \\
1.7\end{array}$ & $\begin{array}{c}\infty \\
10.88 \\
8.42 \\
7.10 \\
6.26 \\
5.65 \\
5.19 \\
4.53\end{array}$ & $\begin{array}{l}1.0059 \\
1.1409 \\
1.1870 \\
1.2273 \\
1.2645 \\
1.2997 \\
1.3336 \\
1.3991\end{array}$ & $\begin{array}{l}1.1377 \\
1.1487 \\
1.1562 \\
1.1637 \\
1.1714 \\
1.1793 \\
1.1872 \\
1.2035\end{array}$ & $\begin{array}{l}1.1812 \\
1.1701 \\
1.1627 \\
1.1554 \\
1.1481 \\
1.1408 \\
1.1336 \\
1.1192\end{array}$ & $\begin{array}{l}0.2691 \\
0.2669 \\
0.2655 \\
0.2640 \\
0.2625 \\
0.2610 \\
0.2595 \\
0.2565\end{array}$ \\
\hline 0.8 & $\begin{array}{l}0.0 \\
0.3 \\
0.5 \\
0.7 \\
0.9 \\
1.1 \\
1.3 \\
1.7\end{array}$ & $\begin{array}{c}\infty \\
10.98 \\
8.55 \\
7.27 \\
6.46 \\
5.88 \\
5.45 \\
4.83\end{array}$ & $\begin{array}{l}1.0155 \\
1.1547 \\
1.2026 \\
1.2448 \\
1.2842 \\
1.3219 \\
1.3586 \\
1.4308\end{array}$ & $\begin{array}{l}1.3373 \\
1.3526 \\
1.3630 \\
1.3735 \\
1.3843 \\
1.3952 \\
1.4063 \\
1.4292\end{array}$ & $\begin{array}{l}1.3917 \\
1.3762 \\
1.3659 \\
1.3556 \\
1.3454 \\
1.3353 \\
1.3252 \\
1.3052\end{array}$ & $\begin{array}{l}0.2341 \\
0.2318 \\
0.2303 \\
0.2287 \\
0.2271 \\
0.2256 \\
0.2240 \\
0.2209\end{array}$ \\
\hline 1.6 & $\begin{array}{l}0.0 \\
0.3 \\
0.5 \\
0.7 \\
0.9 \\
1.1 \\
1.3 \\
1.7\end{array}$ & $\begin{array}{c}\infty \\
11.51 \\
9.39 \\
8.41 \\
7.77 \\
7.21 \\
6.70 \\
5.87\end{array}$ & $\begin{array}{l}1.0301 \\
1.1769 \\
1.2303 \\
1.2804 \\
1.3299 \\
1.3795 \\
1.4291 \\
1.5283\end{array}$ & $\begin{array}{l}1.7447 \\
1.7707 \\
1.7886 \\
1.8068 \\
1.8254 \\
1.8445 \\
1.8640 \\
1.9044\end{array}$ & $\begin{array}{l}1.7954 \\
1.7691 \\
1.7519 \\
1.7347 \\
1.7177 \\
1.7007 \\
1.6839 \\
1.6506\end{array}$ & $\begin{array}{l}0.1849 \\
0.1824 \\
0.1808 \\
0.1791 \\
0.1775 \\
0.1758 \\
0.1742 \\
0.1708\end{array}$ \\
\hline 3.2 & $\begin{array}{l}0.0 \\
0.3 \\
0.5 \\
0.7 \\
0.9 \\
1.1 \\
1.3 \\
1.7\end{array}$ & $\begin{array}{c}\infty \\
15.69 \\
13.22 \\
11.20 \\
9.82 \\
8.82 \\
8.05 \\
6.92\end{array}$ & $\begin{array}{l}1.0427 \\
1.2166 \\
1.2987 \\
1.3767 \\
1.4520 \\
1.5263 \\
1.6007 \\
1.7523\end{array}$ & $\begin{array}{l}2.5611 \\
2.6175 \\
2.6567 \\
2.6971 \\
2.7389 \\
2.7820 \\
2.8267 \\
2.9207\end{array}$ & $\begin{array}{l}2.5972 \\
2.5417 \\
2.5052 \\
2.4692 \\
2.4336 \\
2.3984 \\
2.3636 \\
2.2948\end{array}$ & $\begin{array}{l}0.1300 \\
0.1274 \\
0.1256 \\
0.1239 \\
0.1221 \\
0.1203 \\
0.1185 \\
0.1150\end{array}$ \\
\hline 6.4 & $\begin{array}{l}0.0 \\
0.5 \\
0.7 \\
0.9 \\
1.1 \\
1.3 \\
1.7\end{array}$ & $\begin{array}{c}\infty \\
16.75 \\
13.98 \\
12.17 \\
10.85 \\
9.84 \\
8.35\end{array}$ & $\begin{array}{l}1.1146 \\
1.4389 \\
1.5621 \\
1.6855 \\
1.8120 \\
1.9434 \\
2.2276\end{array}$ & $\begin{array}{l}4.1931 \\
4.4557 \\
4.5706 \\
4.6918 \\
4.8199 \\
4.9555 \\
5.2518\end{array}$ & $\begin{array}{l}4.1980 \\
4.9572 \\
4.8644 \\
4.7729 \\
4.6832 \\
4.5947 \\
4.4216\end{array}$ & $\begin{array}{l}0.0815 \\
0.0769 \\
0.0751 \\
0.0732 \\
0.0713 \\
0.0694 \\
0.0657\end{array}$ \\
\hline \multicolumn{7}{|c|}{$\alpha^{p}=99 \%$} \\
\hline 0.1 & $\begin{array}{l}0.0 \\
0.1 \\
0.5 \\
0.9 \\
1.3 \\
1.7 \\
2.1\end{array}$ & $\begin{array}{c}\infty \\
67.84 \\
30.29 \\
22.55 \\
18.73 \\
16.36 \\
14.70\end{array}$ & $\begin{array}{l}1.0083 \\
1.0277 \\
1.0528 \\
1.0699 \\
1.0822 \\
1.0938 \\
1.1044\end{array}$ & $\begin{array}{l}1.0159 \\
1.0161 \\
1.0170 \\
1.0179 \\
1.0188 \\
1.0197 \\
1.0207\end{array}$ & $\begin{array}{l}1.0070 \\
1.0068 \\
1.0058 \\
1.0049 \\
1.0040 \\
1.0031 \\
1.0022\end{array}$ & $\begin{array}{l}0.9893 \\
0.9892 \\
0.9892 \\
0.9892 \\
0.9891 \\
0.9891 \\
0.9890\end{array}$ \\
\hline
\end{tabular}


APPENDIX V (Continued)

\begin{tabular}{|c|c|c|c|c|c|c|}
\hline \multicolumn{7}{|c|}{$\alpha^{P}=99 \%$} \\
\hline$\frac{2 a}{\eta}$ & $\alpha_{0}(G)$ & $G$ & $S_{c}$ & $K_{c}^{P}$ & $\hat{r}_{c}$ & $x_{0}^{\prime r}$ \\
\hline \multirow[t]{7}{*}{0.2} & 0.0 & $x$ & 1.0323 & 1.0754 & 1.0440 & 0.9862 \\
\hline & 0.1 & 67.84 & 1.0525 & 1.0757 & 1.0438 & 0.9862 \\
\hline & 0.5 & 30.30 & 1.0789 & 1.0766 & 1.0428 & 0.9861 \\
\hline & 0.9 & 22.55 & 1.0959 & 1.0777 & 1.0418 & 0.9861 \\
\hline & 1.3 & 18.74 & 1.1098 & 1.0787 & 1.0407 & 0.9860 \\
\hline & 1.7 & 16.36 & 1.1221 & 1.0797 & 1.0397 & 0.9860 \\
\hline & 2.1 & 14.70 & 1.1332 & 1.0808 & 1.0387 & 0.9859 \\
\hline \multirow[t]{7}{*}{0.4} & 0.0 & 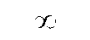 & 1.1049 & 1.3131 & 1.2155 & 0.9700 \\
\hline & 0.1 & 67.84 & 1.1282 & 1.3135 & 1.2151 & 0.9700 \\
\hline & 0.5 & 30.31 & 1.1586 & 1.3150 & 1.2137 & 0.9699 \\
\hline & 0.9 & 22.56 & 1.1783 & 1.3165 & 1.2123 & 0.9697 \\
\hline & 1.3 & 18.75 & 1.1944 & 1.3180 & 1.2109 & 0.9696 \\
\hline & 1.7 & 16.38 & 1.2086 & 1.3195 & 1.2095 & 0.9695 \\
\hline & 2.1 & 14.72 & 1.2215 & 1.3211 & 1.2081 & 0.9694 \\
\hline \multirow[t]{7}{*}{0.8} & 0.0 & $x$ & 1.2370 & 1.8459 & 1.5668 & 0.9175 \\
\hline & 0.1 & 67.86 & 1.2670 & 1.8466 & 1.5662 & 0.9174 \\
\hline & 0.5 & 30.34 & 1.3055 & 1.8496 & 1.5638 & 0.9171 \\
\hline & 0.9 & 22.61 & 1.3305 & 1.8526 & 1.5613 & 0.9167 \\
\hline & 1.3 & 1881 & 1.3512 & 1.8556 & 1.5589 & 0.9164 \\
\hline & 1.7 & 16.44 & 1.3694 & 1.8587 & 1.5565 & 0.9161 \\
\hline & 2.1 & 14.79 & 1.3861 & 1.8617 & 1.5541 & 0.9157 \\
\hline \multirow[t]{7}{*}{1.6} & 0.0 & $\alpha$ & 1.4039 & 2.7650 & 2.0849 & 0.8109 \\
\hline & 0.1 & 67.92 & 1.4447 & 2.7667 & 2.0836 & 0.8107 \\
\hline & 0.5 & 30.48 & 1.4951 & 2.7733 & 2.0788 & 0.8100 \\
\hline & 0.9 & 22.81 & 1.5283 & 2.7801 & 2.0740 & 0.8092 \\
\hline & 1.3 & 19.05 & 1.5559 & 2.7869 & 2.0692 & 0.8084 \\
\hline & 1.7 & 16.73 & 1.5805 & 2.7938 & 2.0644 & 0.8076 \\
\hline & 2.1 & 15.12 & 1.6031 & 2.8007 & 2.0595 & 0.8069 \\
\hline \multirow[t]{7}{*}{3.2} & 0.0 & $x$ & 1.5757 & 4.4021 & 2.9524 & 0.6487 \\
\hline & 0.1 & 68.17 & 1.6317 & 4.4063 & 2.9495 & 0.6484 \\
\hline & 0.5 & 31.12 & 1.6969 & 4.4233 & 2.9384 & 0.6469 \\
\hline & 0.9 & 23.79 & 1.7412 & 4.4405 & 2.9274 & 0.6455 \\
\hline & 1.3 & 20.43 & 1.7792 & 4.4579 & 2.9163 & 0.6441 \\
\hline & 1.7 & 18.53 & 1.8144 & 4.4755 & 2.9053 & 0.6426 \\
\hline & 2.1 & 17.34 & 1.8482 & 4.4933 & 2.8943 & 0.6412 \\
\hline \multirow[t]{7}{*}{6.4} & 0.0 & $x$ & 1.7213 & 7.5122 & 4.5859 & 0.4583 \\
\hline & 0.1 & 69.29 & 1.7988 & 7.5245 & 4.5782 & 0.4577 \\
\hline & 0.5 & 35.75 & 1.8837 & 7.5742 & 4.5486 & 0.4556 \\
\hline & 0.9 & 32.01 & 1.9524 & 7.6247 & 4.5193 & 0.4534 \\
\hline & 1.3 & 29.42 & 2.0203 & 7.6761 & 4.4897 & 0.4512 \\
\hline & 1.7 & 26.39 & 2.0864 & 7.7284 & 4.4604 & 0.4489 \\
\hline & 2.1 & 23.82 & 2.1497 & 7.7816 & 4.4312 & 0.4467 \\
\hline
\end{tabular}


APPENDIX VI-INSTRUMENTAL CORRECTION FACTORS FOR LORENTZ LINES EXPECTED FROM A SPECTROMETER HAVING A COMBINATION SLIT FUNCTION, $\sigma_{\boldsymbol{g}^{3}}\left(v, v^{\prime}\right)$

\begin{tabular}{|c|c|c|c|c|c|c|}
\hline & & & $P=30^{\circ}$ & & & \\
\hline$\frac{2 a}{\gamma}$ & $\alpha_{0}(G)$ & $G$ & $S_{c}$ & $K_{c}^{P}$ & $\gamma_{c}$ & $\alpha_{0}^{\prime P}$ \\
\hline 0.1 & 0.0 & $\infty$ & 1.0002 & 1.0005 & 1.0068 & 0.2988 \\
\hline & 0.3 & 10.85 & 1.1323 & 1.0133 & 0.9983 & 0.2967 \\
\hline & 0.5 & 8.38 & 1.1775 & 1.0191 & 0.9928 & 0.2953 \\
\hline & 0.7 & 7.06 & 1.2169 & 1.0250 & 0.9872 & 0.2939 \\
\hline & 0.9 & 6.20 & 1.2532 & 1.0310 & 0.9817 & 0.2925 \\
\hline & 1.1 & 5.59 & 1.2874 & 1.0370 & 0.9762 & 0.2910 \\
\hline & 1.3 & 5.13 & 1.3202 & 1.0432 & 0.9706 & 0.2896 \\
\hline & 1.7 & 4.45 & 1.3833 & 1.0557 & 0.9595 & 0.2867 \\
\hline 0.2 & 0.0 & $\infty$ & 1.0008 & 1.0172 & 1.0243 & 0.2958 \\
\hline & 0.3 & 10.85 & 1.1330 & 1.0260 & 1.0157 & 0.2936 \\
\hline & 0.5 & 8.38 & 1.1782 & 1.0320 & 1.0099 & 0.2922 \\
\hline & 0.7 & 7.06 & 1.2177 & 1.0380 & 1.0042 & 0.2908 \\
\hline & 0.9 & 6.21 & 1.2541 & 1.0442 & 0.9985 & 0.2894 \\
\hline & 1.1 & 5.59 & 1.2883 & 1.0504 & 0.9928 & 0.2879 \\
\hline & 1.3 & 5.13 & 1.3213 & 1.0567 & 0.9872 & 0.2865 \\
\hline & 1.7 & 4.46 & 1.3845 & 1.0695 & 0.9759 & 0.2836 \\
\hline 0.4 & 0.0 & $\infty$ & 1.0015 & 1.0567 & 1.0769 & 0.2865 \\
\hline & 0.3 & 10.86 & 1.1352 & 1.0662 & 1.0676 & 0.2843 \\
\hline & 0.5 & 8.39 & 1.1807 & 1.0726 & 1.0613 & 0.2829 \\
\hline & 0.7 & 7.07 & 1.2205 & 1.0791 & 1.0552 & 0.2814 \\
\hline & 0.9 & 6.22 & 1.2570 & 1.0858 & 1.0491 & 0.2800 \\
\hline & 1.1 & 5.61 & 1.2916 & 1.0925 & 1.0429 & 0.2785 \\
\hline & 1.3 & 5.15 & 1.3248 & 1.0993 & 1.0368 & 0.2771 \\
\hline & 1.7 & 4.48 & 1.3887 & 1.1132 & 1.0247 & 0.2741 \\
\hline 0.8 & 0.0 & $\infty$ & 1.0063 & 1.1650 & 1.2123 & 0.2637 \\
\hline & 0.3 & 10.89 & 1.1425 & 1.1765 & 1.2009 & 0.2615 \\
\hline & 0.5 & 8.43 & 1.1888 & 1.1843 & 1.1934 & 0.2600 \\
\hline & 0.7 & 7.13 & 1.2294 & 1.1923 & 1.1860 & 0.2586 \\
\hline & 0.9 & 6.28 & 1.2669 & 1.2004 & 1.1786 & 0.2571 \\
\hline & 1.1 & 5.68 & 1.3024 & 1.2086 & 1.1712 & 0.2556 \\
\hline & 1.3 & 5.23 & 1.3367 & 1.2170 & 1.1637 & 0.2540 \\
\hline & 1.7 & 4.57 & 1.4030 & 1.2341 & 1.1491 & 0.2510 \\
\hline 1.6 & 0.0 & $\infty$ & 1.0160 & 1.4317 & 1.5372 & 0.2205 \\
\hline & 0.3 & 11.06 & 1.1581 & 1.4492 & 1.5203 & 0.2182 \\
\hline & 0.5 & 8.72 & 1.2068 & 1.4611 & 1.5092 & 0.2166 \\
\hline & 0.7 & 9.51 & 1.2506 & 1.4733 & 1.4980 & 0.2150 \\
\hline & 0.9 & 6.69 & 1.2917 & 1.4856 & 1.4870 & 0.2134 \\
\hline & 1.1 & 6.08 & 1.3313 & 1.4982 & 1.4759 & 0.2118 \\
\hline & 1.3 & 5.62 & 1.3698 & 1.5111 & 1.4649 & 0.2102 \\
\hline & 1.7 & 4.93 & 1.4452 & 1.5375 & 1.4431 & 0.2070 \\
\hline 3.2 & 0.0 & $\infty$ & 1.0284 & 2.0335 & 2.2653 & 0.1609 \\
\hline & 0.3 & 12.43 & 1.1865 & 2.0689 & 2.2321 & 0.1584 \\
\hline & 0.5 & 9.91 & 1.2449 & 2.0933 & 2.2101 & 0.1567 \\
\hline & 0.7 & 8.53 & 1.2981 & 2.1183 & 2.1883 & 0.1550 \\
\hline & 0.9 & 7.62 & 1.3488 & 2.1440 & 2.1666 & 0.1533 \\
\hline & 1.1 & 6.97 & 1.3983 & 2.1703 & 2.1451 & 0.1515 \\
\hline & 1.3 & 6.46 & 1.4474 & 2.1974 & 2.1237 & 0.1498 \\
\hline & 1.7 & 5.71 & 1.5458 & 2.2538 & 2.0811 & 0.1464 \\
\hline
\end{tabular}


APPENDIX VI (Continued)

\begin{tabular}{|c|c|c|c|c|c|c|}
\hline$\frac{2 a}{\gamma}$ & $\alpha_{0}(G)$ & $G$ & $\begin{array}{c}\alpha^{P}=30 \\
S_{c}\end{array}$ & $K_{c}^{P}$ & $\gamma_{c}$ & $\alpha_{0}^{\prime P}$ \\
\hline \multirow[t]{2}{*}{6.4} & $\begin{array}{l}0.0 \\
0.3 \\
0.5 \\
0.7 \\
0.9 \\
1.1 \\
1.3 \\
1.7\end{array}$ & $\begin{array}{c}x \\
15.12 \\
12.26 \\
10.67 \\
9.60 \\
8.81 \\
8.20 \\
7.27\end{array}$ & $\begin{array}{l}1.0672 \\
1.2354 \\
1.3141 \\
1.3889 \\
1.4627 \\
1.5373 \\
1.6134 \\
1.7732\end{array}$ & $\begin{array}{l}3.3049 \\
3.3995 \\
3.4658 \\
3.5350 \\
3.6070 \\
3.6823 \\
3.7609 \\
3.9291\end{array}$ & $\begin{array}{l}3.8062 \\
3.7213 \\
3.6658 \\
3.6105 \\
3.5559 \\
3.5013 \\
3.4473 \\
3.3401\end{array}$ & $\begin{array}{l}0.1023 \\
0.0996 \\
0.0978 \\
0.0960 \\
0.0942 \\
0.0923 \\
0.0905 \\
0.0868\end{array}$ \\
\hline & \multicolumn{5}{|c|}{$\alpha^{P}=60 \%$} & \\
\hline 0.1 & $\begin{array}{l}0.0 \\
0.3 \\
0.5 \\
0.7 \\
0.9 \\
1.1 \\
1.3 \\
1.7\end{array}$ & $\begin{array}{r}\propto \\
17.44 \\
13.48 \\
11.38 \\
10.02 \\
9.05 \\
8.31 \\
7.24\end{array}$ & $\begin{array}{l}1.0006 \\
1.0792 \\
1.1044 \\
1.1259 \\
1.1452 \\
1.1629 \\
1.1796 \\
1.2108\end{array}$ & $\begin{array}{l}1.0048 \\
1.0081 \\
1.0103 \\
1.0126 \\
1.0148 \\
1.0171 \\
1.0194 \\
1.0240\end{array}$ & $\begin{array}{l}1.0061 \\
1.0027 \\
1.0006 \\
0.9984 \\
0.9963 \\
0.9941 \\
0.9919 \\
0.9876\end{array}$ & $\begin{array}{l}0.5983 \\
0.5771 \\
0.5962 \\
0.5954 \\
0.5946 \\
0.5938 \\
0.5930 \\
0.5913\end{array}$ \\
\hline 0.2 & $\begin{array}{l}0.0 \\
0.3 \\
0.5 \\
0.7 \\
0.9 \\
1.1 \\
1.3 \\
1.7\end{array}$ & $\begin{array}{c}x \\
17.44 \\
13.49 \\
11.38 \\
10.02 \\
9.05 \\
8.31 \\
7.25\end{array}$ & $\begin{array}{l}1.0021 \\
1.0809 \\
1.1062 \\
1.1278 \\
1.1471 \\
1.1649 \\
1.1817 \\
1.2130\end{array}$ & $\begin{array}{l}1.0178 \\
1.0212 \\
1.0235 \\
1.0258 \\
1.0282 \\
1.0305 \\
1.0328 \\
1.0376\end{array}$ & $\begin{array}{l}1.0224 \\
1.0190 \\
1.0167 \\
1.0145 \\
1.0122 \\
1.0099 \\
1.0077 \\
1.0032\end{array}$ & $\begin{array}{l}0.5935 \\
0.5923 \\
0.5915 \\
0.5907 \\
0.5893 \\
0.5890 \\
0.5882 \\
0.5865\end{array}$ \\
\hline 0.4 & $\begin{array}{l}0.0 \\
0.3 \\
0.5 \\
0.7 \\
0.9 \\
1.1 \\
1.3 \\
1.7\end{array}$ & $\begin{array}{c}x \\
17.44 \\
13.49 \\
11.39 \\
10.03 \\
9.06 \\
8.32 \\
7.26\end{array}$ & $\begin{array}{l}1.0074 \\
1.0866 \\
1.1123 \\
1.1341 \\
1.1536 \\
1.1717 \\
1.1887 \\
1.2205\end{array}$ & $\begin{array}{l}1.0604 \\
1.0641 \\
1.0666 \\
1.0691 \\
1.0716 \\
1.0741 \\
1.0767 \\
1.0819\end{array}$ & $\begin{array}{l}1.0724 \\
1.0687 \\
1.0663 \\
1.0639 \\
1.0615 \\
1.0590 \\
1.0566 \\
1.0517\end{array}$ & $\begin{array}{l}0.5786 \\
0.5773 \\
0.5765 \\
0.5756 \\
0.5747 \\
0.5739 \\
0.5730 \\
0.5713\end{array}$ \\
\hline 0.8 & $\begin{array}{l}0.0 \\
0.3 \\
0.5 \\
0.7 \\
0.9 \\
1.1 \\
1.3 \\
1.7\end{array}$ & $\begin{array}{r}\infty \\
17.46 \\
13.52 \\
11.42 \\
10.07 \\
9.10 \\
8.37 \\
7.31\end{array}$ & $\begin{array}{l}1.0283 \\
1.1035 \\
1.1300 \\
1.1526 \\
1.1729 \\
1.1916 \\
1.2093 \\
1.2424\end{array}$ & $\begin{array}{l}1.1798 \\
1.1844 \\
1.1874 \\
1.1905 \\
1.1937 \\
1.1968 \\
1.2000 \\
1.2064\end{array}$ & $\begin{array}{l}1.2044 \\
1.1999 \\
1.1969 \\
1.1939 \\
1.1909 \\
1.1879 \\
1.1849 \\
1.1790\end{array}$ & $\begin{array}{l}0.5401 \\
0.5387 \\
0.5376 \\
0.5368 \\
0.5359 \\
0.5349 \\
0.5340 \\
0.5321\end{array}$ \\
\hline
\end{tabular}


APPENDIX VI (Continued)

\begin{tabular}{|c|c|c|c|c|c|c|}
\hline & & & $\alpha^{P}=60$ & & & \\
\hline $\begin{array}{c}2 a \\
y\end{array}$ & $a_{0}(G)$ & $G$ & $S_{c}$ & $K_{c}^{P}$ & $\gamma_{c}$ & $\alpha_{0}^{\prime P}$ \\
\hline 1.6 & 0.0 & $\infty$ & 1.0566 & 1.4777 & 1.5256 & 0.4621 \\
\hline & 0.3 & 17.55 & 1.1383 & 1.4849 & 1.5186 & 0.4605 \\
\hline & 0.5 & 13.64 & 1.1668 & 1.4897 & 1.5141 & 0.4594 \\
\hline & 0.7 & 11.57 & 1.1911 & 1.4946 & 1.5095 & 0.4583 \\
\hline & 0.9 & 10.25 & 1.2131 & 1.4996 & 1.5049 & 0.4572 \\
\hline & 1.1 & 9.33 & 1.2336 & 1.5045 & 1.5004 & 0.4561 \\
\hline & 1.3 & 8.64 & 1.2532 & 1.5096 & 1.4958 & 0.4550 \\
\hline & 1.7 & 7.66 & 1.2902 & 1.5197 & 1.4867 & 0.4528 \\
\hline 3.2 & 0.0 & $\infty$ & 1.0827 & 2.1539 & 2.2507 & 0.3465 \\
\hline & 0.3 & 18.11 & 1.1903 & 2.1693 & 2.2368 & 0.3445 \\
\hline & 0.5 & 14.86 & 1.2234 & 2.1797 & 2.2275 & 0.3432 \\
\hline & 0.7 & 12.84 & 1.2529 & 2.1902 & 2.2182 & 0.3419 \\
\hline & 0.9 & 11.47 & 1.2802 & 2.2008 & 2.2090 & 0.3406 \\
\hline & 1.1 & 10.48 & 1.3059 & 2.2115 & 2.1999 & 0.3392 \\
\hline & 1.3 & 9.72 & 1.3305 & 2.2224 & 2.1907 & 0.3379 \\
\hline & 1.7 & 8.61 & 1.3778 & 2.2445 & 2.1722 & 0.3352 \\
\hline 6.4 & 0.0 & $\infty$ & 1.1196 & 3.5857 & 3.7897 & 0.2255 \\
\hline & 0.3 & 21.94 & 1.2538 & 3.6288 & 3.7537 & 0.2231 \\
\hline & 0.5 & 17.71 & 1.2982 & 3.6579 & 3.7296 & 0.2216 \\
\hline & 0.7 & 15.40 & 1.3382 & 3.6875 & 3.7051 & 0.2200 \\
\hline & 0.9 & 13.87 & 1.3759 & 3.7176 & 3.6813 & 0.2184 \\
\hline & 1.1 & 12.76 & 1.4122 & 3.7484 & 3.6575 & 0.2169 \\
\hline & 1.3 & 11.90 & 1.4476 & 3.7797 & 3.6334 & 0.2153 \\
\hline & 1.7 & 10.63 & 1.5172 & 3.8441 & 3.5861 & 0.2121 \\
\hline & & & $=$ & & & \\
\hline
\end{tabular}

\begin{tabular}{|c|c|c|c|c|c|}
\hline 0.0 & $\infty$ & 1.0028 & 1.0052 & 1.0022 & 0.9898 \\
\hline 0.1 & 67.84 & 1.0220 & 1.0054 & 1.0020 & 0.9897 \\
\hline 0.5 & 30.29 & 1.0468 & 1.0063 & 1.0011 & 0.9897 \\
\hline 0.9 & 22.55 & 1.0629 & 1.0072 & 1.0002 & 0.9897 \\
\hline 1.3 & 18.73 & 1.0759 & 1.0081 & 0.9994 & 0.9896 \\
\hline 1.7 & 16.36 & 1.0874 & 1.0090 & 0.9985 & 0.9896 \\
\hline 2.1 & 14.70 & 1.0978 & 1.0099 & 0.9976 & 0.9895 \\
\hline 0.0 & $\infty$ & 1.0113 & 1.0237 & 1.0121 & 0.9889 \\
\hline 0.1 & 67.84 & 1.0307 & 1.0239 & 1.0119 & 0.9889 \\
\hline 0.5 & 30.30 & 1.0560 & 1.0248 & 1.0110 & 0.9888 \\
\hline 0.9 & 22.55 & 1.0723 & 1.0257 & 1.0101 & 0.9888 \\
\hline 1.3 & 18.73 & 1.0856 & 1.0266 & 1.0092 & 0.9887 \\
\hline 1.7 & 16.36 & 1.0973 & 1.0276 & 1.0083 & 0.9887 \\
\hline 2.1 & 14.70 & 1.1079 & 1.0285 & 1.0074 & 0.9886 \\
\hline 0.0 & $\infty$ & 1.0423 & 1.1080 & 1.0693 & 0.9843 \\
\hline 0.1 & 67.84 & 1.0630 & 1,1082 & 1.0690 & 0.9843 \\
\hline 0.5 & 30.30 & 1.0900 & 1.1093 & 1.0680 & 0.9843 \\
\hline 0.9 & 22.55 & 1.1073 & 1.1104 & 1.0669 & 0.9842 \\
\hline 1.3 & 18.74 & 1.1215 & 1.1115 & 1.0658 & 0.9841 \\
\hline 1.7 & 16.37 & 1.1340 & 1.1125 & 1.0647 & 0.9841 \\
\hline 2.1 & 14.70 & 1.1454 & 1.1136 & 1.0637 & 0.9840 \\
\hline
\end{tabular}


APPENDIX VI (Continued)

\begin{tabular}{|c|c|c|c|c|c|c|}
\hline & & & $P=99^{\circ}$ & & & \\
\hline$\frac{2 a}{\gamma}$ & $\alpha_{0}(G)$ & $G$ & $S_{r}$ & $K_{r}^{P}$ & $\gamma_{s}$ & $\alpha_{0}^{\prime P}$ \\
\hline 0.8 & 0.0 & $\infty$ & 1.1217 & 1.3662 & 1.2478 & 0.9656 \\
\hline & 0.1 & 67.85 & 1.1463 & 1.3667 & 1.2474 & 0.9656 \\
\hline & 0.5 & 30.31 & 1.1777 & 1.3683 & 1.2459 & 0.9655 \\
\hline & 0.9 & 22.57 & 1.1980 & 1.3699 & 1.2444 & 0.9653 \\
\hline & 1.3 & 18.76 & 1.2147 & 1.3716 & 1.2428 & 0.9652 \\
\hline & 1.7 & 16.39 & 1.2294 & 1.3732 & 1.2413 & 0.9650 \\
\hline & 2.1 & 14.73 & 1.2428 & 1.3749 & 1.2398 & 0.9649 \\
\hline 1.6 & 0.0 & $\infty$ & 1.2586 & 1.9332 & 1.6190 & 0.9076 \\
\hline & 0.1 & 67.87 & 1.2915 & 1.9341 & 1.6182 & 0.9076 \\
\hline & 0.5 & 30.36 & 1.3315 & 1.9373 & 1.6155 & 0.9072 \\
\hline & 0.9 & 22.63 & 1.3576 & 1.9406 & 1.6129 & 0.9068 \\
\hline & 1.3 & 18.84 & 1.3791 & 1.9439 & 1.6101 & 0.9064 \\
\hline & 1.7 & 16.48 & 1.3982 & 1.9473 & 1.6074 & 0.9060 \\
\hline & 2.1 & 14.83 & 1.4156 & 1.9506 & 1.6047 & 0.9057 \\
\hline 3.2 & 0.0 & $\infty$ & 1.4350 & 3.0961 & 2.3666 & 0.7740 \\
\hline & 0.1 & 67.95 & $1: 4820$ & 3.0982 & 2.3650 & 0.7738 \\
\hline & 0.5 & 30.56 & 1.5351 & 3.1066 & 2.3590 & 0.7729 \\
\hline & 0.9 & 22.95 & 1.5703 & 3.1150 & 2.3529 & 0.7720 \\
\hline & 1.3 & 19.28 & 1.5997 & 3.1236 & 2.3470 & 0.7711 \\
\hline & 1.7 & 17.10 & 1.6262 & 3.1322 & 2.3409 & 0.7701 \\
\hline & 2.1 & 15.60 & 1.6509 & 3.1409 & 2.3347 & 0.7692 \\
\hline 6.4 & 0.0 & $\propto$ & 1.6113 & 5.4788 & 3.9078 & 0.5685 \\
\hline & 0.1 & 68.31 & 1.6801 & 5.4853 & 3.9035 & 0.5681 \\
\hline & 0.5 & 32.62 & 1.7503 & 5.5117 & 3.8870 & 0.5664 \\
\hline & 0.9 & 25.88 & 1.8012 & 5.5384 & 3.8709 & 0.5646 \\
\hline & 1.3 & 22.01 & 1.8454 & 5.5655 & 3.8541 & 0.5628 \\
\hline & 1.7 & 19.56 & 1.8859 & 5.5929 & 3.8379 & 0.5611 \\
\hline & 2.1 & 17.82 & 1.9241 & 5.6207 & 3.8214 & 0.5593 \\
\hline
\end{tabular}

